\title{
D-branes and normal functions
}

\author{
David R. Morrison ${ }^{1,2}$ and Johannes Walcher $^{3}$
}

${ }^{1}$ Center for Geometry and Theoretical Physics, Duke University, Durham, NC 27708, USA

${ }^{2}$ Departments of Mathematics and Physics, University of California, Santa Barbara, CA 93106, USA

${ }^{3}$ School of Natural Sciences, Institute for Advanced Study, Princeton, NJ 08540, USA

\begin{abstract}
We explain the B-model origin of extended Picard-Fuchs equations satisfied by the D-brane superpotential on compact Calabi-Yau threefolds. The domainwall tension is identified with a Poincaré normal function - a transversal holomorphic section of the Griffiths intermediate Jacobian - via the Abel-Jacobi map. Within this formalism, we derive the extended Picard-Fuchs equation associated with the mirror of the real quintic.
\end{abstract}

Contents

1 Introduction

2 Normal functions and D-branes 
2.2 Abel-Jacobi map on the derived category 561

2.3 Comments on open problems $\quad 563$

3 The real quintic and its mirror $\quad 565$

3.1 Six hundred and twenty-five real quintics $\quad 565$

$\begin{array}{lll}3.2 & \text { The prediction } & 566\end{array}$

3.3 Matrix factorization $\quad 568$

3.4 Intersection index $\quad 570$

3.5 Bundles $\quad 572$

3.6 From matrix factorization to curve 575

4 Main computation $\quad 576$

$\begin{array}{lll}\text { 4.1 Sketch of computation } & 577\end{array}$

4.2 Resolution of singularities $\quad 578$

4.3 Inhomogeneous Picard-Fuchs via Griffiths-Dwork $\quad 580$

4.4 Boundary conditions and monodromy $\quad 585$

5 Summary and conclusions $\quad 586$

$\begin{array}{ll}\text { Acknowledgments } & 587\end{array}$

$\begin{array}{ll}\text { Appendix } & 587\end{array}$

References $\quad 594$

\section{Introduction}

Mirror symmetry is a powerful tool to manipulate physical and mathematical data associated with Calabi-Yau manifolds. Soon after the earliest examples of mirror symmetry [1-3], a computation of the special geometry and the enumeration of rational curves on the quintic were made by Candelas et al. [4]. The computation was explained Hodge theoretically in [5] and the 
verification of the enumerative predictions was completed in [6,7]. Approaches to derive mirror symmetry from the worldsheet point of view have also been discussed $[8,9]$.

Meanwhile, D-branes have entered mirror symmetry in a variety of ways. To name the most important, Witten [10] showed that for open topological strings, cubic string field theory reduces to ordinary or holomorphic ChernSimons theory. Kontsevich [11] proposed to understand mirror symmetry as an equivalence of $A_{\infty}$-categories, whose objects were later identified as D-branes. Strominger et al. [12] used D-branes to develop the geometric picture of mirror symmetry as a duality of torus fibrations. Vafa and various collaborators (beginning with Gopakumar) have shown that Bogomol'nyi Prasad-Sommerfield (BPS) states of D-branes are extremely useful invariants that carry a lot of physical and enumerative information [13]. Douglas [14] has complemented the picture by a general formulation of stability conditions on D-brane categories (see also [15]).

In the course of these developments, the established theory underlying closed string mirror symmetry for Calabi-Yau manifolds - special geometry and Gromov-Witten invariants - has played a very useful supporting role. It has, however, not always been clear whether D-branes would ultimately be part of the traditional picture or how one would derive the closed string story, e.g., from D-brane categories. (This problem was posed already in [11]; for some recent work, see [16-18].) As a physicist, one feels that in some sense, the underlying reason is that $A_{\infty}$-categories are too big. Since D-brane categories are defined off-shell, they carry a lot of redundant, gaugedependent information. With some hindsight, one is led to ask the natural question: What is the invariant physical information stored in the derived category?

In this paper, we give answers to these questions by picking up the Hodge theoretic considerations. Our main motivation is the recent realization that at least in some cases, there is indeed invariant information in the open string sector beyond its cohomology. Walcher [19] showed that for a certain D-brane configuration on the quintic, ${ }^{1}$ the on-shell value of the superpotential, as a function over closed string moduli space, satisfies a differential equation which is an extension of the Picard-Fuchs equation which governs closed string mirror symmetry. According to general principles, this superpotential makes enumerative predictions in the A-model, which were subsequently verified rigorously in [22]. In this work, we will explain the B-model origin of this extended Picard-Fuchs equation. Previous studies of analogous problems in local Calabi-Yau manifolds include [23,24], whose

\footnotetext{
${ }^{1}$ Very similar results appear to hold for many other one-parameter models [20, 21].
} 
enumerative predictions were verified in $[25,26]$ and whose differential equations were discussed in $[27,28]$ (see also $[29,30]$ ).

The main idea to derive Picard-Fuchs equations in the context of open strings has been implicit in many previous works. Consider, for simplicity, the case when we are wrapping a D5-brane on a curve in some second homology class of our Calabi-Yau manifold. Assume that this class has two isolated holomorphic representatives $C_{+}$and $C_{-}$. Choose a three-chain $\Gamma$, $\partial \Gamma=C_{+}-C_{-}$connecting these two representatives. $C_{+}$and $C_{-}$correspond physically to two supersymmetric vacua of an $\mathcal{N}=1$ supersymmetric theory on the brane worldvolume. The tension of a BPS domainwall between the two vacua is, $\mathcal{T}=\mathcal{W}_{+}-\mathcal{W}_{-}$, equal to the superpotential difference, and is given by the geometric formula [31]

$$
\mathcal{W}_{+}(z)-\mathcal{W}_{-}(z)=\mathcal{T}(z)=\int_{\Gamma} \Omega(z),
$$

where $\Omega(z)$ is the holomorphic three-form as a function of complex structure moduli.

The Picard-Fuchs equation, $\mathcal{L} \Pi(z)=0$, is the (in general, system of partial) differential equation satisfied by any period $\Pi(z)=\int_{\Gamma^{\mathrm{c}}} \Omega(z)$ of the holomorphic three-form over a closed three-cycle, $\partial \Gamma^{\mathrm{c}}=0$. When applying the Picard-Fuchs operator to a chain integral as in (1.1), we will not, in general, obtain zero. One type of non-vanishing contribution arises as a boundary term, but there are, in general, also other terms from differentiating the chain $\Gamma$. The inhomogeneous Picard-Fuchs equation associated with $C_{+}-C_{-}$is then

$$
\mathcal{L} \mathcal{T}(z)=f(z) .
$$

As we will review below, this inhomogeneous equation is well-defined by the algebraic cycle $C_{+}-C_{-}$and does not depend on the choice of chain $\Gamma$. It is also known (although we will not review this) that $f(z)$ is necessarily a rational function of the suitable algebraic coordinate on moduli space.

The general existence of inhomogeneous Picard-Fuchs equations similar to (1.2) has been known in the mathematical literature at least as early as [32]. (In dimension 1, of course, such notions are completely classical.) A fairly recent reference with examples worked out in dimension 2 (i.e., for K3 surfaces) is [33]. The main result of the present work is a complete and mathematically rigorous derivation of the inhomogeneous Picard-Fuchs equation satisfied by $\mathcal{T}(z)$ for the B-brane mirror to the real quintic. This is, to our knowledge, the first explicit example of an inhomogeneous PicardFuchs equation in dimension bigger than 2 . 
The particular form of the inhomogeneous Picard-Fuchs equation for the real quintic was originally guessed in [19] based on very restrictive monodromy properties that its solution should possess. Combined with the results of [22], our derivation puts open string mirror symmetry for the real quintic at an equal level with the classical mirror theorems on rational curves in Calabi-Yau three-folds.

Before doing the computation in Section 4 (some details having been deferred to the appendix), we will describe in Section 2 how normal functions and the variation of mixed Hodge structure capture certain invariant information of the open string sector. We will not attempt a detailed comparison with the local toric case $[27,28]$. It would be very interesting to understand better the relation between those works and ours, especially with regard to open string moduli. We also note that the insights into the relation between D-branes and normal functions have proven central in the recent computation of loop amplitudes in the open topological string using the extended holomorphic anomaly equation [34].

In Section 3, we review in a self-contained manner the geometry of the real quintic and its mirror. This will help explain some of the original background that led to the extended Picard-Fuchs equation. Alternatively, one can view our results in this paper as further evidence for the conjectural relation between the real quintic(s) and certain objects in the derived category of the mirror quintic. This could be a starting point for establishing homological mirror symmetry for the quintic. We present our conclusions in Section 5.

\section{Normal functions and D-branes}

The urge to understand the differential equation of [19] in Hodge theoretic terms is very natural. In hindsight, it is not even surprising that the correct framework is the theory of Poincaré normal functions, applied to Calabi-Yau three-folds. That theory was developed by Griffiths $[32,35]$ as an integral part of Hodge theory in higher dimension. Picard-Fuchs equations play an important role in the variation of Hodge structure and have been central to mirror symmetry for closed strings. So one should naturally have wondered about the use of normal functions in this context.

On the other hand, there are very good reasons to believe that normal functions will not be the full story for open string mirror symmetry computations. As is now well accepted, D-branes on Calabi-Yau manifolds can only be fully understood in some sophisticated categorical framework. The D-brane superpotential, which is the physical observable governed by the 
differential equation, is realized mathematically in a fairly complicated way in the framework of $A_{\infty}$ categories $[36,37]$. From this point of view, the relevance of classical Hodge theory is not immediate at all.

The purpose of this section is to compile the main definitions and theorems pertaining to normal functions, as well as to explain, to the best of our present understanding, the relation to the D-brane superpotential. We point out that our main computation in Section 4 takes this general theory as useful background, but does not, strictly speaking, depend on it.

\subsection{Normal functions attached to algebraic cycles}

For more details on normal functions, we recommend Griffiths' original papers $[32,35]$, as well as the books $[38,39]$. For an introduction to Hodge theory, see [40].

Let $\left(H_{\mathbb{Z}}^{2 k-1}, F^{*} H_{\mathbb{C}}^{2 k-1}\right)$ be an integral Hodge structure of odd weight $2 k-1$. The Griffiths intermediate Jacobian is the complex torus

$$
J^{2 k-1}=\frac{H_{\mathbb{C}}^{2 k-1}}{F^{k} H_{\mathbb{C}}^{2 k-1} \oplus H_{\mathbb{Z}}^{2 k-1}} .
$$

As real torus, $J^{2 k-1}$ is isomorphic to $H_{\mathbb{R}}^{2 k-1} / H_{\mathbb{Z}}^{2 k-1}$, and the complex structure on $J^{2 k-1}$ arises from the identification $H_{\mathbb{C}}^{2 k-1} / F^{k} H_{\mathbb{C}}^{2 k-1} \cong H_{\mathbb{R}}^{2 k-1}$ as real vector spaces. Now if $\left(H_{\mathbb{Z}}^{2 k-1}, F^{*} \mathcal{H}^{2 k-1}\right)$ is an integral variation of Hodge structure of weight $2 k-1$ over some base $M$, we can consider a relative version of $(2.1)$,

$$
\mathcal{J}^{2 k-1}=\frac{\mathcal{H}^{2 k-1}}{F^{k} \mathcal{H}^{2 k-1} \oplus H_{\mathbb{Z}}^{2 k-1}} .
$$

$\mathcal{J}^{2 k-1} \rightarrow M$ is known as the Griffiths intermediate Jacobian fibration of the integral variation of Hodge structure.

A normal function of the variation of Hodge structure is a holomorphic section $\nu$ of the intermediate Jacobian fibration (2.2) satisfying Griffiths transversality for normal functions ${ }^{2}$

$$
\nabla \tilde{\nu} \in F^{k-1} \mathcal{H}^{2 k-1} \otimes \Omega_{M}^{1},
$$

\footnotetext{
${ }^{2}$ We are here omitting the regularity conditions on normal functions that are required when the variation of Hodge structure degenerates. Those will play only a minor role in our application.
} 
where $\tilde{\nu}$ is any lift of $\nu$ to $\mathcal{H}^{2 k-1}$. Also, $\nabla$ is the Gauss-Manin connection and $\Omega_{M}^{1}$ the sheaf of differentials on $M$. It is easy to see that the condition (2.3) is independent of the lift. For if $\tilde{\nu}^{\prime}$ is another lift, then

$$
\tilde{\nu}^{\prime}=\tilde{\nu}+\eta_{F}+\eta_{\mathbb{Z}}
$$

where $\eta_{F}$ is a section of $F^{k} \mathcal{H}^{2 k-1}$ and $\eta_{\mathbb{Z}}$ is a section of $H_{\mathbb{Z}}^{2 k-1}$. The claim follows since $\nabla \eta_{\mathbb{Z}}=0$ and $\nabla \eta_{F} \in F^{k-1} \mathcal{H}^{2 k-1} \otimes \Omega_{M}^{1}$ by Griffiths transversality applied to $\mathcal{H}^{2 k-1}$.

The variation of Hodge structure of interest in this paper arises from the deformation of complex structure of a family $\mathcal{Y} \rightarrow M$ of Calabi-Yau three-folds with typical fiber $Y$. The interesting values of $k$ in this case are $k=1,2$, and 3 . The intermediate Jacobians for $k=1, J^{1}$ and for $k=3, J^{5}$ are known as the Picard variety and the Albanese variety, respectively. But when $Y$ is simply connected, $J^{1}=J^{5}=0$, and the only interesting value is $k=2,2 k-1=3$.

In the geometric situation, let us say in dimension $n$, not necessarily equal to 3 , a useful source of normal functions are the homologically trivial algebraic cycles. Let $\mathcal{C} \in \mathcal{Z}^{k}(\mathcal{Y})$ be a relative algebraic cycle of codimension $k$, flat over $M$, i.e., $\mathcal{C}=\sum n_{i} \mathcal{C}^{i}$ is a finite integral linear combination of algebraic subsets of $\mathcal{Y}$. This cycle is "homologically trivial," denoted $\mathcal{C} \in$ $\mathcal{Z}^{k}(\mathcal{Y})_{\text {hom }}$ if the image of $C_{m}=\mathcal{C} \cap Y_{m}$ in $H^{2 k}\left(Y_{m} ; \mathbb{Z}\right)$ is trivial for all $m \in$ $M$. (Here, $Y_{m}$ is the fiber of $\mathcal{Y} \rightarrow M$ over $m \in M$, and we are associating codimension $k$ cycles with $(k, k)$-forms via Poincaré duality.)

Before defining the normal function, we note that in the geometric situation, we have the isomorphism $(n:=\operatorname{dim}(Y))$

$$
J^{2 k-1}\left(Y_{m}\right)=\left(F^{n-k+1} H^{2 n-2 k+1}\left(Y_{m}\right)\right)^{*} / H_{2 n-2 k-1}\left(Y_{m} ; \mathbb{Z}\right),
$$

which follows from the isomorphism $H^{2 k-1} / F^{k} H^{2 k-1} \cong\left(F^{n-k+1} H^{2 n-2 k+1}\right)^{*}$, and the equivalence from Poincare duality, $\left(H^{2 k-1}(Y ; \mathbb{Z})\right)^{*} \cong H_{2 n-2 k+1}$ $(Y ; \mathbb{Z})$, given by integration.

Stepping on (2.5), to define the normal function associated with $\mathcal{C}$, we need to specify a map $\nu_{\mathcal{C}}: F^{n-k+1} \mathcal{H}^{2 n-2 k+1} \rightarrow \mathcal{O}_{M}$, defined modulo periods $H_{2 n-2 k+1}(Y ; \mathbb{Z})$. To this end, for each $m \in M$, we pick a $2 n-2 k+1$-chain $\Gamma_{m}$ such that

$$
\partial \Gamma_{m}=C_{m} \quad \text { in } Y_{m}
$$

where $C_{m}=\mathcal{C} \cap Y_{m}$ as a codimension- $k$ cycle. Such a chain exists because $C_{m}$ is homologically trivial, but is ambiguous by closed $2 n-2 k+1$ cycles. 
If we require that $\Gamma_{m}$ depend in a continuous fashion on $m$, the ambiguity is reduced to $H_{2 n-2 k+1}(Y ; \mathbb{Z})$.

Now given $[\omega] \in F^{n-k+1} \mathcal{H}^{2 n-2 k+1}$, we can locally on $M$ representing it by a relative $2 n-2 k+1$-form $\omega \in F^{n-k+1} \mathcal{A}^{2 n-2 k+1}$ that is closed in the fiber direction and well defined up to the image of $d^{Y}: F^{n-k+1} \mathcal{A}^{2 n-2 k} \rightarrow$ $F^{n-k+1} \mathcal{A}^{2 n-2 k+1}$ (this last assertion follows from the Dolbeault theorem). We then define

$$
\nu_{\mathcal{C}}([\omega])_{m}:=\int_{\Gamma_{m}} \omega_{m}
$$

Let us check that this is well defined. If we choose a different representative $\omega^{\prime}$ of $[\omega]$, the difference is

$$
\int_{\Gamma_{m}}\left(\omega_{m}^{\prime}-\omega_{m}\right)=\int_{\partial \Gamma_{m}} \alpha_{m}
$$

where $\alpha \in F^{n-k+1} \mathcal{A}^{2 n-2 k}$. This vanishes by type considerations since $\partial \Gamma_{m}=$ $C_{m}$ is holomorphic, so Poincaré dual to a $(k, k)$-form.

Finally, we check holomorphicity and transversality. Namely, we analyze the variation of (2.7) as $m$ varies to first order in $M$. If $v$ is a (not necessarily holomorphic) complexified tangent vector to $M$ at $m$, Kodaira-Spencer theory provides us with a lift, $v^{\prime}$, of $v$ to $T \mathcal{Y}$. The differential of (2.7) in the direction of $v$ can be written as

$$
d_{v}\left(\nu_{\mathcal{C}}([\omega])\right)_{m}=-\int_{C_{m}}\left(\omega_{m}, v^{\prime}\right)+\int_{\Gamma_{m}}\left(\tilde{\nabla}_{v} \omega\right)_{m},
$$

where $\tilde{\nabla}_{v} \omega$ represents $\nabla_{v}[\omega]$ and $\nabla$ is the Gauss-Manin connection on $\mathcal{H}^{2 n-2 k+1}$.

To check holomorphicity, we let $v$ be anti-holomorphic and $[\omega]$ be a holomorphic section of $F^{n-k+1} \mathcal{H}^{2 n-2 k+1}$. We then have that $\nabla_{v}[\omega]=0$ in $\mathcal{H}^{2 n-2 k+1}$. In fact, $\left(\tilde{\nabla}_{v} \omega\right)_{m}=d^{Y}\left(\omega_{m}, v^{\prime}\right)$ by Kodaira-Spencer. Thus, (2.9) vanishes, and $\nu_{\mathcal{C}}$ is a holomorphic section of $\mathcal{J}^{2 k-1}$.

To show transversality, we take $v$ to be holomorphic. Note that the statement $\nabla_{v} \tilde{\nu}_{\mathcal{C}} \in F^{k-1} \mathcal{H}^{2 k-1}$ is under the isomorphism $H^{2 k-1} / F^{k-1} H^{2 k-1} \cong$ $\left(F^{n-k+2} H^{2 n-2 k+1}\right)^{*}$ (see $\left.(2.5)\right)$ equivalent to the assertion that $\left(\nabla_{v} \tilde{\nu}_{\mathcal{C}}\right)([\omega])_{m}$ $=0$ for $[\omega] \in F^{n-k+2} \mathcal{H}^{2 n-2 k+1}$, where $\tilde{\nu}_{\mathcal{C}}$ is any lift of $\nu_{\mathcal{C}}$ to $\left(\mathcal{H}^{2 n-2 k+1}\right)^{*}$. By the compatibility of the Gauss-Manin connection with Poincaré duality, $d_{v}\left(\tilde{\nu}_{\mathcal{C}}([\omega])\right)_{m}=\left(\nabla_{v} \tilde{\nu}_{\mathcal{C}}\right)([\omega])_{m}+\tilde{\nu}_{\mathcal{C}}\left(\nabla_{v}[\omega]\right)_{m}$, so this criterion becomes $d_{v}\left(\tilde{\nu}_{\mathcal{C}}([\omega])\right)_{m}=\tilde{\nu}_{\mathcal{C}}\left(\nabla_{v}[\omega]\right)_{m}$, which is already independent of the lift. Now 
if $[\omega] \in F^{n-k+2} \mathcal{H}^{2 n-2 k+1},\left(\omega_{m}, v^{\prime}\right) \in F^{n-k+1} \mathcal{H}^{2 n-2 k}$, the first term in (2.9) vanishes by type consideration. This implies transversality.

We close this subsection with one more definition: The association

$$
\text { AJ: } \mathcal{Z}^{k}(\mathcal{Y})_{\text {hom }} \rightarrow \mathcal{J}^{2 k-1}(\mathcal{Y}), \quad \mathcal{C} \mapsto \nu_{\mathcal{C}}
$$

is known as the Abel-Jacobi map. It contains some useful information about algebraic cycles and their algebraic equivalences. The theory is particularly rich for Calabi-Yau three-folds (as mentioned above, the interesting value is then $k=2$ ) and led to a lot of early results on questions related to holomorphic curves $[35,41,42]$. That subject was later revolutionized by mirror symmetry and Gromov-Witten theory. As we will try to convey in this article, normal functions are returning to the enterprise as well, with promising applications in the context of D-branes and mirror symmetry for open strings.

\subsection{Abel-Jacobi map on the derived category}

To explain the relevance of normal functions to D-branes, in general, we take as starting point Witten's holomorphic Chern-Simons functional. We denote by $Y$ a (compact) Calabi-Yau three-fold, $E$ a holomorphic vector bundle over $Y$, with $\bar{\partial}$ the Dolbeault operator coupled to $E$. If $a \in$ $A^{(0,1)}(Y, \operatorname{End}(E))$ is a $(0,1)$-form with values in the endomorphisms of $E$, we define

$$
S_{\mathrm{hCS}}(a)=\int_{Y} \operatorname{Tr}\left(\frac{1}{2} a \wedge \bar{\partial} a+\frac{1}{3} a \wedge a \wedge a\right) \wedge \Omega,
$$

where $\Omega$ is the (unique up to scale) holomorphic $(3,0)$-form on $Y$. The functional (2.11) was originally proposed in [43], as an expression for the space-time superpotential in the context of the heterotic string. This proposal can be explained on the basis that the critical points of (2.11) are precisely those $a \in A^{(0,1)}(Y, \operatorname{End}(E))$ for which the $(0,2)$-part of the curvature vanishes,

$$
F^{(0,2)}=\bar{\partial} a+a \wedge a=0,
$$

i.e., the deformed operator $\bar{\partial}_{a}=\bar{\partial}+a$ is an alternative Dolbeault operator on $E$, viewed as a differentiable vector bundle on $Y$. In general, $\bar{\partial}_{a}$ will define a different complex structure on $E$.

Witten [10] showed that, in the context of the topological string, the functional (2.11) is the target space or string field theory action describing 
the tree-level dynamics of open strings on $Y$ coupled to $E$ (a topological B-brane). This also led to the suggestion that holomorphic Chern-Simons should make sense as a quantum theory, and to various puzzles related to non-renormalizability of (2.11), appearance of closed strings as intermediate states, etc. The classical theory has been analyzed in depth over the years (see [44] for a review). The recent results on open-closed topological string [34] can be viewed as giving partial answers to the problems related to the quantum theory.

Connections of the holomorphic Chern-Simons functional with the theory of normal functions have appeared in the mathematical literature in [45, 46] (see also [47]). In the physics literature, a relation to the Abel-Jacobi map for curves on Calabi-Yau three-fold was established, e.g., in [23,4850]. When our B-brane, instead of being specified by a holomorphic vector bundle, is wrapping a holomorphic curve $C$, it was shown in $[23,49,50]$ that the dimensional reduction of the holomorphic Chern-Simons action is nothing but the Abel-Jacobi integral

$$
S(C)=\int_{\Gamma} \Omega \quad \text { with } \partial \Gamma=C-C_{0}
$$

viewed as a functional on all possible curves homotopic to some given reference holomorphic curve $C_{0}$.

Neglecting the dynamics of open strings, the most direct physical interpretation of the formulas (2.11) and (2.13) is as the tension of BPS domainwalls connecting the background vacuum $\left(\bar{\partial}\right.$ or $\left.C_{0}\right)$, on the D-brane worldvolume, with some other vacuum, corresponding to a non-trivial critical point, $a_{*}$ or $C_{*}$, respectively.

$$
\mathcal{T}=\left\{\begin{array}{l}
\mathcal{W}\left(a_{*}\right)-\mathcal{W}(0)=S_{\mathrm{hCS}}\left(a_{*}\right) \\
\mathcal{W}\left(C_{*}\right)-\mathcal{W}\left(C_{0}\right)=S\left(C_{*}\right)
\end{array}\right.
$$

It should be clear that, even neglecting open string dynamics, those expressions cannot be fully satisfactory for describing the superpotential for an arbitrary B-brane, which might be neither a holomorphic vector bundle nor a holomorphic curve in general. The algebraic device needed to generalize these formulas to an arbitrary object $B$ in $D^{b}(Y)$ (or some category equivalent to it) is the notion of the algebraic second Chern class, $c_{2}^{\mathrm{alg}}(B)$ [51]. It takes values in the Chow group $\mathrm{CH}^{2}(Y)$ of algebraic cycles of codimension 2, modulo rational equivalence. The image of $c_{2}^{\text {alg }}(B)$ in cohomology $H^{4}(Y ; \mathbb{Z})$ is equal to the ordinary (topological) second Chern class $c_{2}^{\text {top }}(B)$, but $c_{2}^{\text {alg }}$ is generally a more refined invariant. 
The algebraic Chern class satisfies axioms very similar to its topological counterpart. In particular, it splits exact triangles in the D-brane category. If

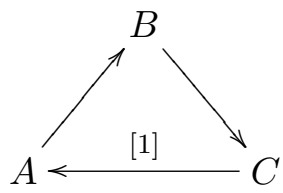

for three objects, $A, B$, and $C$, then the total Chern classes $c^{\text {alg }}=1+\sum c_{i}^{\text {alg }}$ satisfy

$$
c^{\mathrm{alg}}(A) \cdot c^{\operatorname{alg}}(C)=c^{\mathrm{alg}}(B),
$$

which together with functoriality (and its behavior on holomorphic line bundles) is essentially enough to define $c^{\text {alg }}$.

Using the algebraic second Chern class puts us directly in the situation discussed in the previous subsection. When $c_{2}^{\text {top }}(B)=0 \in H^{4}(Y ; \mathbb{Z})$, the algebraic cycle defined by $c_{2}^{\text {alg }}(B) \in \mathrm{CH}^{2}(Y)$ is homologically trivial and yields, for fixed $Y$, an Abel-Jacobi class according to the above discussion. When $B$ suitably deforms with $Y$, we obtain a normal function $\nu_{B}=\nu_{c_{2}^{\text {alg }}(B)}$. In particular, the formula for the domainwall tension is

$$
\mathcal{T}=\nu_{B}(\Omega)
$$

where $\Omega$ is the same holomorphic three-form as above. It is not hard to see that this definition reduces to (2.11) and (2.13) when $B$ is a holomorphic vector bundle or a holomorphic curve, respectively.

We should emphasize that the second Chern class will certainly not capture all the intricacies of the superpotential for a general B-brane on a Calabi-Yau. This will require a much more sophisticated analysis, partly along the lines of the cited literature.

\subsection{Comments on open problems}

Before turning to the applications, we will collect a few more remarks from the general theory of normal functions, some of which might prove valuable for further developments.

\section{Extension of Hodge structure}

Let $\left(H_{\mathbb{Z}}^{2 k-1}, F^{*} \mathcal{H}^{2 k-1}\right)$ be an integral variation of Hodge structure of weight $2 k-1$ over a base $M$. Let $\nu$ be a normal function. In Exercises 1 and 2 
in Chapter 7 of [39], it is shown that this data can be used to define an extension of Hodge structure to yield an integral variation of mixed Hodge structure. At the integral level, this is a locally trivial extension

$$
H_{\mathbb{Z}}^{2 k-1} \rightarrow\left(H_{\mathbb{Z}}=H_{\mathbb{Z}}^{2 k-1} \oplus \mathbb{Z}\right) \rightarrow \mathbb{Z}
$$

The weight filtration is given by $W_{2 k-2} H_{\mathbb{Z}}=0, W_{2 k-1} H_{\mathbb{Z}}=H_{\mathbb{Z}}^{2 k-1}, W_{2 k}=$ $H_{\mathbb{Z}}$, whereas the Hodge filtration on $\mathcal{H}=\left(H_{\mathbb{Z}}^{2 k-1} \oplus \mathbb{Z}\right) \otimes \mathcal{O}_{M}$ is such that it reduces to the given Hodge filtration $F^{*} \mathcal{H}^{2 k-1}$ on $\mathcal{H}^{2 k-1}$, and to $F^{k+1} \mathcal{O}_{M}=$ $0, F^{k} \mathcal{O}_{M}=\mathcal{O}_{M}$ on the quotient.

In the context of mirror symmetry, a different mixed Hodge structure is relevant. This mixed Hodge structure is associated with the degeneration at a point of maximal unipotent monodromy in the moduli space $[5,52]$. The monodromy calculations of [19], partially reviewed in Section 4, are indicative of a very interesting interaction between this limiting mixed Hodge structure and the one given by extension using the normal function (2.18). It would be interesting to elucidate this further.

\section{More extensions?}

We have so far largely suppressed the existence of an $A_{\infty}$-structure on the category of B-branes, except to ask the natural question how much of that structure is possibly captured by the normal function? In thinking about this problem, we are led to the following speculations.

The $A_{\infty}$-structure on a brane $B$ in the category of $\mathrm{B}$-branes is given by a collection of "higher" products $m_{n}$ satisfying certain conditions of associativity. At the level of the string worldsheet, the $m_{n}$ with $n \geq 2$ can be determined by computing the (topological) disk amplitudes with $n+1$ open string insertions on the boundary. $m_{1}$ is identified with the open string BRST operator. Finally, $m_{0}$ is related to the bulk-to-boundary obstruction map by taking one derivative with respect to the closed string moduli [34].

From general considerations, as well as the identification of the disk amplitude with two bulk insertions as the Griffiths infinitesimal invariant [34], it appears natural that the normal function $\nu$ can fit as an " $m_{-1}$ " into the $A_{\infty}$-structure. As emphasized in [34], the obstruction map can be interpreted Hodge theoretically as the dual of the infinitesimal Abel-Jacobi map. Those two observations suggest that one should try to understand whether the higher $A_{\infty}$ products $m_{n}$ for $n \geq 1$ can also be given a Hodge theoretic interpretation. 


\section{A-model version}

All considerations in this paper are phrased in the language of the B-model. On the other hand, we recall that much of the deeper understanding of classical (closed string) mirror symmetry involved the reconstruction of Hodge theoretic structures in the A-model. In particular, the importance of quantum cohomology and the structure of the mirror map become especially clear in the "A-model variation of Hodge structure" [53,54].

It would be very interesting to extend these insights to the open string. A general definition of a functional conjecturally mirror to the holomorphic Chern-Simons functional/domainwall tension (see equation (2.14)) is given in [22], extending [10]. This functional includes corrections from worldsheet instantons (holomorphic disks ending on Lagrangian submanifolds) and should in principle be related to Floer theory and the Fukaya category, as the open string analogues of quantum cohomology. This relation should be similar to that between the holomorphic Chern-Simons functional and the derived category. In the A-model, the precise relation is not currently understood, but as an intermediate step, it would be interesting to check at least the Hodge theoretic statements pertaining to normal functions, based on, say, axioms for open Gromov-Witten invariants.

\section{The real quintic and its mirror}

Our interest now turns to the quintic Calabi-Yau $X=\{G=0\} \subset \mathbb{P}^{4}$, defined as the vanishing locus of a degree 5 polynomial $G$ in five complex variables $x_{1}, \ldots, x_{5}$. We assume that $X$ is defined over the reals, which means that all coefficients of $G$ are real (possibly up to some common phase). The real locus $\left\{x_{i}=\bar{x}_{i}\right\} \subset X$ is then a Lagrangian submanifold, and after choosing a flat $U(1)$ connection, will define an object in the (derived) Fukaya category $\operatorname{Fuk}(X)$. In this section, we will first review a proposal which identifies a mirror object in the category of B-branes of the mirror quintic, in its LandauGinzburg description. Via some detours, we will be able to derive from the matrix factorization the corresponding normal function. In the next section, we will then show by an explicit computation that this normal function satisfies precisely the inhomogeneous Picard-Fuchs equation proposed in [19].

\subsection{Six hundred and twenty-five real quintics}

Both the topological type and the homology class in $H_{3}(X ; \mathbb{Z})$ of the real locus depend on the complex structure of $X$ (the choice of (real) polynomial 
$G)$. On the other hand, the Fukaya category is independent of the choice of $G$ (real or not). The object in $\operatorname{Fuk}(X)$ that we shall refer to as the real quintic is defined from the real locus $L$ of $X$ when $G$ is the Fermat quintic $G=x_{1}^{5}+x_{2}^{5}+x_{3}^{5}+x_{4}^{5}+x_{5}^{5}$. It is not hard to see that topologically $L \cong \mathbb{R P}^{3}$. There are therefore two choices of flat bundles on $L$, and we will denote the corresponding objects of $\operatorname{Fuk}(X)$ by $L_{+}$and $L_{-}$, respectively. More precisely, since Fuk $(X)$ depends on the choice of a complexified Kähler structure on $X$, we define $L_{ \pm}$for some choice of Kähler parameter $t$ close to large volume $\operatorname{Im}(t) \rightarrow \infty$, and then continue it under Kähler deformations. In fact, the rigorous definition of the Fukaya category is at present only known infinitesimally close to this large volume point [55]. However, $\operatorname{Fuk}(X)$ does exist over the entire stringy Kähler moduli space of $X$, and at least some of the structures vary holomorphically. Our interest here is in the variation of the categorical structure associated with $L_{ \pm}$over the entire stringy Kähler moduli space of $X$, identified via mirror symmetry with the complex structure moduli space of the mirror quintic $Y$.

The Fermat quintic is invariant under more than one anti-holomorphic involution. If $\mathbb{Z}_{5}$ denotes the multiplicative group of fifth roots of unity, we define for $\chi=\left(\chi_{1}, \ldots, \chi_{5}\right) \in\left(\mathbb{Z}_{5}\right)^{5}$ an anti-holomorphic involution $\sigma_{\chi}$ of $\mathbb{P}^{4}$ by its action on homogeneous coordinates

$$
\sigma_{\chi}: x_{i} \rightarrow \chi_{i} \bar{x}_{i} .
$$

The Fermat quintic is invariant under any $\sigma_{\chi}$. The involution and the fixedpoint locus only depend on the class of $\chi$ in $\left(\mathbb{Z}_{5}\right)^{5} / \mathbb{Z}_{5} \cong\left(\mathbb{Z}_{5}\right)^{4}$, and we obtain in this way $5^{4}=625$ (pairs of) objects $L_{ \pm}^{[\chi]}$ in $\operatorname{Fuk}(X)$. We will return to those 625 real quintics below, and for the moment focus on $L_{ \pm}=L_{ \pm}^{[\chi=1]}$.

We emphasize again that although we have defined the Lagrangians $L_{ \pm}^{[\chi]}$ as fixed-point sets of anti-holomorphic involutions of the Fermat quintic, we can think of the corresponding objects of $\operatorname{Fuk}(X)$ without reference to the complex structure.

\subsection{The prediction}

The image in $K^{0}(\operatorname{Fuk}(X))$ is the same for $L_{+}$and $L_{-}$. This is the counterpart in the A-model of the triviality of topological Chern classes $c^{\text {top }}\left(B_{+}-\right.$ $B_{-}$) for two objects $B_{ \pm}$in the category of B-branes. As mentioned above, there should exist a definition of an Abel-Jacobi map to a normal function of the A-model variation of Hodge structure constructed from the quantum cohomology of $X[53,54]$. As explained in [19], this normal function can be 
realized geometrically by wrapping a D-brane on a disk $D$ whose boundary on $L$ represents the non-trivial element of $H_{1}(L ; \mathbb{Z}) \cong \mathbb{Z}_{2}$. Neglecting instanton corrections, the corresponding truncated normal function is $\frac{t}{2} \pm \frac{1}{4} \bmod t \mathbb{Z}+\mathbb{Z} .^{3} \quad$ Instanton corrections deform this to

$$
\mathcal{T}_{A}(t)=\frac{t}{2} \pm\left(\frac{1}{4}+\frac{1}{2 \pi^{2}} \sum_{d \text { odd }} n_{d} q^{d / 2}\right),
$$

where $q=\mathrm{e}^{2 \pi \mathrm{i} t}$, and $n_{d}$ are the open Gromov-Witten invariants of the real quintic defined in [56], predicted in [19], and fully computed in [22]. The precise result for the $n_{d}$ is as follows.

Mirror symmetry for the quintic is governed by the differential operator

$$
\mathcal{L}=\theta^{4}-5 z(5 \theta+1)(5 \theta+2)(5 \theta+3)(5 \theta+4),
$$

where $\theta=z d / d z$. As we will review further below, $\mathcal{L}$ is the Picard-Fuchs operator of the mirror quintic. The equation $\mathcal{L} \varpi(z)=0$ has four linearly independent solutions. Two of those solutions are given by the following power-series expansion around $z=0$ :

$$
\begin{aligned}
& \varpi_{0}(z)=\sum_{m=0}^{\infty} \frac{(5 m) !}{(m !)^{5}} z^{m} \\
& \varpi_{1}(z)=\varpi_{0}(z) \log z+5 \sum_{m=1}^{\infty} \frac{(5 m) !}{(m !)^{5}} z^{m}[\Psi(1+5 m)-\Psi(1+m)],
\end{aligned}
$$

and determine the mirror map as

$$
t=t(z)=\frac{1}{2 \pi \mathrm{i}} \frac{\varpi_{1}(z)}{\varpi_{0}(z)}, \quad q(z)=\exp (2 \pi \mathrm{i} t(z)) .
$$

The result of $[19,22]$ is

$$
\mathcal{L}\left(\varpi_{0}(z) \mathcal{T}_{A}(z)\right)=\frac{15}{16 \pi^{2}} \sqrt{z} .
$$

Combined with the boundary conditions (3.2), this is equivalent to

$$
\varpi_{0}(z) \mathcal{T}_{A}(z)=\frac{\varpi_{1}(z)}{4 \pi \mathrm{i}}+\frac{\varpi_{0}(z)}{4}+\frac{15}{\pi^{2}} \tau(z),
$$

\footnotetext{
${ }^{3}$ The sign depends on whether we consider $L_{+}-L_{-}$or $L_{-}-L_{+}$. For this to make sense, note that $\frac{t}{2}-\frac{1}{4}=-\left(\frac{t}{2}+\frac{1}{4}\right) \bmod t \mathbb{Z}+\mathbb{Z}$. For details, see [19].
} 
where

$$
\tau(z)=\frac{\Gamma(3 / 2)^{5}}{\Gamma(7 / 2)} \sum_{m=0}^{\infty} \frac{\Gamma(5 m+7 / 2)}{\Gamma(m+3 / 2)^{5}} z^{m+1 / 2}=\sqrt{z}+\frac{5005}{9} z^{3 / 2}+\cdots
$$

gives a particular solution of the inhomogeneous Picard-Fuchs equation (3.6).

\subsection{Matrix factorization}

For the rest of this work, $W$ will denote the one-parameter family of quintic polynomials

$$
W=\frac{1}{5}\left(x_{1}^{5}+x_{2}^{5}+x_{3}^{5}+x_{4}^{5}+x_{5}^{5}\right)-\psi x_{1} x_{2} x_{3} x_{4} x_{5} .
$$

Geometrically, the mirror quintic, $Y$, is the quotient of this one-parameter family of quintics by $\left(\mathbb{Z}_{5}\right)^{3}=\left(\mathbb{Z}_{5}\right)^{4} / \mathbb{Z}_{5}$, where $\left(\mathbb{Z}_{5}\right)^{4}$ is the group of phase symmetries of $W$ (for $\psi \neq 0$ ). Alternatively, we can think of a LandauGinzburg orbifold model with worldsheet superpotential $W$ and orbifold group $\left(\mathbb{Z}_{5}\right)^{4}$.

We will also have occasion to work in the B-model on the one-parameter family of quintic hypersurfaces given by $W=0$ in $\mathbb{P}^{4}$ (without quotient). In this context, we will denote this family by $X_{\psi}$. When we work in the context of the A-model, with an arbitrary complex structure represented by a general quintic polynomial $G$, we will continue to denote the quintic simply by $X$.

Recall that for a quintic $X$ defined by $G=0$, the homological CalabiYau/Landau-Ginzburg correspondence [57-60] states that the derived category of coherent sheaves of $X$ is equivalent to the graded, equivariant category of matrix factorizations of the corresponding Landau-Ginzburg superpotential,

$$
D^{b}(X)=\operatorname{MF}\left(G / \mathbb{Z}_{5}\right)
$$

where $\mathbb{Z}_{5}$ is the diagonal group of phase symmetries. The analogous statement for the mirror quintic is

$$
D^{b}(Y) \cong \operatorname{MF}\left(W / \mathbb{Z}_{5}^{4}\right)
$$

with $\left(\mathbb{Z}_{5}\right)^{4}$ as above.

To describe an object mirror to the real quintic, we begin with finding a matrix factorization of the one-parameter family of superpotentials (3.9). 
If $V \cong \mathbb{C}^{5}$ is a five-dimensional vector space, we can associate to its exterior algebra a $\mathbb{C}\left[x_{1}, \ldots, x_{5}\right]$-module $M=\wedge^{*} V \otimes \mathbb{C}\left[x_{1}, \ldots, x_{5}\right]$. It naturally comes with the decomposition

$$
M=M_{0}+M_{1}+M_{2}+M_{3}+M_{4}+M_{5}, \quad M_{s}=\wedge^{s} V \otimes \mathbb{C}\left[x_{1}, \ldots, x_{5}\right],
$$

and the $\mathbb{Z}_{2}$-grading $(-1)^{i}$. Let $\eta_{i}$ be a basis of $V$ and $\bar{\eta}_{i}$ the dual basis of $V^{*}$, both embedded in $\operatorname{End}(M)$. We then define two families of matrix factorizations $\left(M, Q_{ \pm}\right)$of $W$ by

$$
Q_{ \pm}=\frac{1}{\sqrt{5}} \sum_{i=1}^{5}\left(x_{i}^{2} \eta_{i}+x_{i}^{3} \bar{\eta}_{i}\right) \pm \sqrt{\psi} \prod_{i=1}^{5}\left(\eta_{i}-x_{i} \bar{\eta}_{i}\right) .
$$

To check that $Q_{ \pm}^{2}=W \operatorname{id}_{M}$, one uses that $\eta_{i}$ and $\bar{\eta}_{i}$ satisfy the Clifford algebra

$$
\left\{\eta_{i}, \bar{\eta}_{j}\right\}=\delta_{i j}
$$

as well as the ensuing relations

$$
\left\{\left(x_{i}^{2} \eta_{i}+x_{i}^{3} \bar{\eta}_{i}\right),\left(\eta_{i}-x_{i} \bar{\eta}_{i}\right)\right\}=0 \quad \text { and } \quad\left(\eta_{i}-x_{i} \bar{\eta}_{i}\right)^{2}=-x_{i} .
$$

The matrix factorization (3.13) is quasi-homogeneous $\left(\mathbb{C}^{*}\right.$-gradable). The $\mathrm{R}$-charges of the superpotential and the $x_{i}$ are 2 and $2 / 5$, respectively. So if we assign R-charge $1 / 5$ and $-1 / 5$ to $\eta_{i}$ and $\bar{\eta}_{i}$, respectively, $Q$ will have uniform R-charge 1 . Since $Q$ is irreducible, this determines the R-charge of $M$ uniquely up to an overall shift. As explained in [57], this ambiguity should be fixed by $\operatorname{Tr} R=0$ for studying the stability of the matrix factorizations. But for the present purposes, we will use a different convention (see below).

To specify objects in $\operatorname{MF}(W / \Gamma)$, where $\Gamma=\mathbb{Z}_{5}$ or $\left(\mathbb{Z}_{5}\right)^{4}$ for the quintic and mirror quintic, respectively, we have to equip $M$ with a representation of $\Gamma$ such that $Q$ is equivariant with respect to the action of $\Gamma$ on $x_{i}$. Since $Q$ is irreducible, this representation of $\Gamma$ on $M$ is determined up to a character of $\Gamma$ by a representation on $V$, i.e., an action on the $\eta_{i}$. For $\gamma \in \Gamma$, we have $\gamma\left(x_{i}\right)=\gamma_{i} x_{i}$ for some fifth root of unity $\gamma_{i}$. We then set $\gamma\left(\eta_{i}\right)=\gamma_{i}^{-2} \eta_{i}$, making $Q$ equivariant. As noted, this representation is unique up to an action on $M_{0}$, i.e., a character of $\Gamma$.

For the mirror quintic, $\Gamma=\operatorname{Ker}\left(\left(\mathbb{Z}_{5}\right)^{5} \rightarrow \mathbb{Z}_{5}\right)$, so $\Gamma^{*}=\left(\mathbb{Z}_{5}\right)^{5} / \mathbb{Z}_{5}$, and we label its characters as $[\chi]$. The corresponding objects of $\operatorname{MF}(W / \Gamma)$ constructed out of $Q_{ \pm}(3.13)$ are classified as $Q_{ \pm}^{[\chi]}=\left(M, Q_{ \pm}, \rho_{[\chi]}\right)$, where $\rho_{[\chi]}$ is the representation on $M$ we just described. 
Conjecture: There is an equivalence of categories $\operatorname{Fuk}(X) \cong \mathrm{MF}(W /$ $\left.\left(\mathbb{Z}_{5}\right)^{4}\right)$ which identifies the 625 pairs of objects $L_{ \pm}^{[\chi]}$ with the 625 pairs of equivariant matrix factorizations $Q_{ \pm}^{[\chi]}$.

Note: One can formulate a similar conjecture for any hypersurface in weighted projective space which has a Fermat point in its complex structure moduli space.

\subsection{Intersection index}

The first piece of evidence for the above conjecture comes from Ref. [61]. In that paper, the 625 Lagrangian submanifolds of $X$ described above were associated with the so-called $\mathbf{L}=(1,1,1,1,1)$ A-type Recknagel-Schomerus states in the Gepner model. These A-type boundary states had been constructed in [62] as tensor products of Cardy states in the $\mathcal{N}=2$ minimal model building blocks of the Gepner model. In turn, these Cardy states of the minimal model were identified in [63] with the Lagrangian wedge branes of opening angle $4 \pi / 5$ in the Landau-Ginzburg description of the $\mathcal{N}=2$ minimal models. Via mirror symmetry for the minimal models, those wedges are equivalent to the matrix factorizations based on $x_{i}^{5}=x_{i}^{2} x_{i}^{3}$ (see, e.g., [64]). These are precisely the building blocks of the factorization (3.13), specialized to $\psi=0$. The above deformation away from $\psi=0$, as well as the identification of the pairs $Q_{ \pm}^{[\chi]}$ with the pairs of objects $L_{ \pm}^{[\chi]}$, was first noted in [65], following the suggestion of [61].

The initial step in the above identification of $L_{ \pm}^{[\chi]}$ with $Q_{ \pm}^{[\chi]}$ was justified in [61] by a comparison of the intersection indices of the $L_{ \pm}^{[\chi]}$ with the corresponding intersection indices of the Gepner model boundary states. We will reproduce this here using the matrix factorizations. The match of the domainwall tensions ${ }^{4} L_{+}-L_{-}$and $Q_{+}-Q_{-}$computed in the A-model and B-model, respectively, constitutes further evidence for the above conjecture.

Let us start with the geometric intersection index between ${ }^{5} L^{[\chi]}$ and $L^{\left[\chi^{\prime}\right]}$. Because of the projective equivalence, we have to look at the intersection of the fixed-point loci of $\sigma_{\chi}$ and $\sigma_{\omega \chi^{\prime}}$ from (3.1) where $\omega$ runs over the five

\footnotetext{
${ }^{4}$ Note that because of the symmetries, these domainwall tensions do not depend on the discrete group representation.

${ }^{5}$ The intersection index, being topological, does not depend on the Wilson lines on the A-branes. For the B-branes, it is correspondingly independent of the sign of the square root in (3.13).
} 
fifth roots of unity. It is not hard to see that topologically

$$
\operatorname{Fix}\left(\sigma_{\chi}\right) \cap \operatorname{Fix}\left(\sigma_{\omega \chi^{\prime}}\right) \cap X \cong \mathbb{R P}^{d-2}, \quad d=\#\left\{\chi_{i}^{\prime}=\omega \chi_{i}\right\} .
$$

After making the intersection transverse by a small deformation in the normal direction, we obtain a vanishing contribution for $d=0,1,3,5$, and \pm 1 for $d=2,4$, where the sign depends on the non-trivial phase differences $\chi_{i}^{*} \omega \chi_{i}^{\prime}$. Explicitly, one finds

$$
L^{[\chi]} \cap L^{\left[\chi^{\prime}\right]}=\sum_{\omega \in \mathbb{Z}_{5}} f_{1}\left(\chi^{\prime *} \omega \chi\right),
$$

where

$$
f_{1}(\chi)= \begin{cases}\prod_{i=1}^{5} \operatorname{sgn}\left(\operatorname{Im}\left(\chi_{i}\right)\right), & \text { if } \#\left\{i, \chi_{i}=1\right\}=2,4, \\ 0 & \text { else. }\end{cases}
$$

To compute the intersection index between the matrix factorizations, we use the index theorem of [57]. It says in general

$$
\begin{aligned}
\chi \operatorname{Hom}\left((M, Q, \rho),\left(M^{\prime}, Q^{\prime}, \rho^{\prime}\right)\right) & :=\sum_{i}(-1)^{i} \operatorname{dim} \operatorname{Hom}^{i}\left((M, Q, \rho),\left(M^{\prime}, Q^{\prime}, \rho^{\prime}\right)\right) \\
& =\frac{1}{|\Gamma|} \sum_{\gamma \in \Gamma} \operatorname{Str}_{M^{\prime}} \rho^{\prime}(\gamma)^{*} \frac{1}{\prod_{i=1}^{5}\left(1-\gamma_{i}\right)} \operatorname{Str}_{M} \rho(\gamma),
\end{aligned}
$$

where $\gamma_{i}$ are the eigenvalues of $\gamma \in \Gamma$ acting on $x_{i}$, and $\rho$ and $\rho^{\prime}$ are the representations of $\Gamma$ on $M$. For $M=M^{\prime}, Q=Q^{\prime}$, and $\rho=\rho_{[\chi]}$, and $\rho^{\prime}=\rho_{\left[\chi^{\prime}\right]}$ described above, this evaluates to

$$
-\frac{1}{5^{4}} \sum_{\gamma \in\left(\mathbb{Z}_{5}\right)^{4}} \chi\left(\gamma^{\prime}\right)^{*} \chi(\gamma) \prod_{i=1}^{5}\left(\gamma_{i}+\gamma_{i}^{2}-\gamma_{i}^{3}-\gamma_{i}^{4}\right)=-\sum_{\omega \in \mathbb{Z}_{5}} f_{2}\left(\chi^{\prime *} \omega \chi\right)
$$

where

$$
f_{2}(\chi)= \begin{cases}\prod_{i=1}^{5} \operatorname{sgn}\left(\operatorname{Im}\left(\chi_{i}\right)\right), & \text { if } \#\left\{i, \chi_{i}=1\right\}=0 \\ 0 & \text { else. }\end{cases}
$$

We do not know any generally valid result from the representation theory of finite cyclic group which shows that (3.17) and (3.20) coincide. It is, 
however, not hard to check by hand or computer that for all $\chi$,

$$
\sum_{\omega \in \mathbb{Z}_{5}}\left(f_{1}+f_{2}\right)(\omega \chi)=0 .
$$

Hence

$$
L^{[\chi]} \cap L^{\left[\chi^{\prime}\right]}=\chi \operatorname{Hom}\left(Q^{[\chi]}, Q^{\left[\chi^{\prime}\right]}\right)
$$

as claimed.

\subsection{Bundles}

We now proceed with the construction of the normal function from the matrix factorization (3.13). To this end, we use the homological CalabiYau/Landau-Ginzburg correspondence (3.10) for the quintic as described in [60]. This will produce for us a set of five complexes of coherent sheaves (bundles) on the one-parameter family of quintics $X_{\psi}$. By making those equivariant with respect to the geometric $\left(\mathbb{Z}_{5}\right)^{3}$ action (thus implementing (3.11)) will yield the 625 objects in $D^{b}(Y)$ mirror to the real quintics.

The technique underlying the algorithm of [60] is the gauged linear sigma model of [66]. Thus, we first construct a D-brane in the gauged linear sigma model from the equivariant matrix factorization, and in the second step a complex of (line) bundles on the quintic. We have to and can live with two ambiguities in the construction. The first ambiguity is the Landau-Ginzburg monodromy (cyclic permutation of the characters of $\Gamma=\mathbb{Z}_{5}$ ), whereas the second depends on a certain "band restriction rule" for assignment of the gauge charges in the linear sigma model. The upshot of the construction is the following. We can view the matrix factorization, namely the $\mathbb{Z}_{2}$-graded module $M$ equipped with $Q$ of $Q^{2}=W$ as a 2-periodic infinite complex over the affine singularity $W=0$. We then truncate this infinite complex to a semi-infinite complex in a way that depends on the charge and representation assignments in the gauged linear sigma model. The departure of this construction from the traditional (Serre) correspondence between sheaves on the hypersurface and graded modules on the affine singularity is that the cohomological grading of the complexes also depends on the linear sigma model charges. We now implement this algorithm in our example, referring to [60] for the complete details.

Given $\left(M, Q, \rho_{\chi}\right)$, we first assign R-charges (i.e., a $\mathbb{C}^{*}$-representation, generated by a rational Hermitian matrix, $R$ on $M$ ) in such a way that

$$
\mathrm{e}^{\mathrm{i} \pi R}=\rho_{\chi}(\gamma)(-1)^{s},
$$


where $(-1)^{s}$ is the $\mathbb{Z}_{2}$-grading on $M$ and $\gamma \equiv \mathrm{e}^{2 \pi \mathrm{i} / 5}$ is the generator of $\mathbb{Z}_{5}$. In the decomposition $(3.12), \rho_{\chi}(\gamma)=\mathrm{e}^{2 \pi \mathrm{i}(n-2 s) / 5}$, where $\chi=\mathrm{e}^{2 \pi \mathrm{i} n / 5}$. We choose the R-charge assignment of $M_{s}$ in (3.12) as $R_{s}=\frac{s}{5}+\frac{2 n}{5}$.

Following the algorithm of [60], we now select a "band" of five consecutive integers $\Lambda=\{0,1,2,3,4\}$ and find for each $s$ an integer $\tilde{R}_{s}=s \bmod 2$ and an integer $q_{s} \in \Lambda$ such that

$$
R_{s}=\tilde{R}_{s}-\frac{2 q_{s}}{5}
$$

$\tilde{R}_{s}$ and $q_{s}$ are uniquely determined by this equation. Depending on $n$, we find for the pairs $\left(\tilde{R}_{s}, q_{s}\right)$ the following table:

\begin{tabular}{|c|c|c|c|c|c|}
\hline$n$ & 0 & 1 & 2 & 3 & 4 \\
\hline 0 & $(0,0)$ & $(2,4)$ & $(2,3)$ & $(2,2)$ & $(2,1)$ \\
\hline 1 & $(1,2)$ & $(1,1)$ & $(1,0)$ & $(3,4)$ & $(3,3)$ \\
\hline 2 & $(2,4)$ & $(2,3)$ & $(2,2)$ & $(2,1)$ & $(2,0)$ \\
\hline 3 & $(1,1)$ & $(1,0)$ & $(3,4)$ & $(3,3)$ & $(3,2)$ \\
\hline 4 & $(2,3)$ & $(2,2)$ & $(2,1)$ & $(2,0)$ & $(4,4)$ \\
\hline 5 & $(1,0)$ & $(3,4)$ & $(3,3)$ & $(3,2)$ & $(3,1)$ \\
\hline
\end{tabular}

This data yields a graded, gauge-invariant matrix factorization, $Q_{\mathrm{GLSM}}$ of the linear sigma model superpotential $W_{\mathrm{GLSM}}=P W$, where $P$ is Witten's P-field [66]. In reducing to the non-linear sigma model on the hypersurface, the bulk modes of $P$ are integrated out, while the quantization of the single boundary degree of freedom yields the Fock space of a harmonic oscillator, $\mathcal{H}^{P} \cong \oplus_{N \geq 0} \mathcal{H}_{N}^{P}$, where each $\mathcal{H}_{N}^{P} \cong \mathbb{C}$. The resulting complex on the quintic hypersurface is built from the tensor product $M \otimes \mathcal{H}^{P}$, where $M_{s} \otimes \mathcal{H}_{N}^{P}$ is placed in homological degree $d=\tilde{R}_{s}+2 N$ and twisted by the line bundle $\mathcal{O}\left(q_{s}+5 N\right)$. The original matrix factorization $Q$ acts on this complex in a way compatible with all gradings.

For the data above, we obtain explicitly the following five complexes (here, $V^{s} \equiv \wedge^{s} V$, and the integer in square brackets indicates the homological degree of the first term in the complex):

$$
\begin{aligned}
& n=0:[0] \\
& \mathcal{O}(0) \otimes V^{0} \quad \mathcal{O}(2) \otimes V^{1} \quad \mathcal{O}(5) \otimes V^{0} \quad \mathcal{O}(7) \otimes V^{1}
\end{aligned}
$$

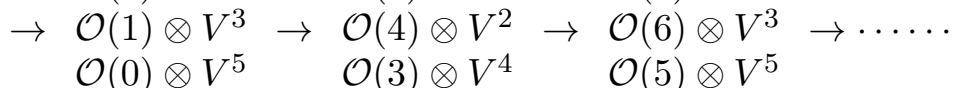


$n=1:[1]$

$$
\begin{aligned}
& \mathcal{O}(1) \otimes V^{1} \quad \mathcal{O}(4) \otimes V^{0} \quad \mathcal{O}(6) \otimes V^{1} \quad \mathcal{O}(9) \otimes V^{0} \\
& \begin{aligned}
\mathcal{O}(0) \otimes V^{3} \rightarrow & \mathcal{O}(3) \otimes V^{2} \\
& \mathcal{O}(2) \otimes V^{4}
\end{aligned} \rightarrow \begin{array}{l}
\mathcal{O}(5) \otimes V^{3} \\
\mathcal{O}(4) \otimes V^{5}
\end{array} \rightarrow \begin{array}{c}
\mathcal{O}(8) \otimes V^{2} \\
\mathcal{O}(7) \otimes V^{4}
\end{array} \rightarrow \cdots \cdots
\end{aligned}
$$

$n=2:[1]$

$$
\begin{aligned}
& \mathcal{O}(0) \otimes V^{1} \quad \mathcal{O}(3) \otimes V^{0} \quad \mathcal{O}(5) \otimes V^{1} \quad \mathcal{O}(8) \otimes V^{0} \\
& \rightarrow \mathcal{O}(2) \otimes V^{2} \rightarrow \mathcal{O}(4) \otimes V^{3} \rightarrow \mathcal{O}(7) \otimes V^{2} \\
& \mathcal{O}(1) \otimes V^{4} \quad \mathcal{O}(3) \otimes V^{5} \quad \mathcal{O}(6) \otimes V^{4}
\end{aligned}
$$

$n=3:[2]$

$$
\begin{aligned}
& \mathcal{O}(2) \otimes V^{0} \\
& \mathcal{O}(1) \otimes V^{2} \\
& \mathcal{O}(0) \otimes V^{4}
\end{aligned} \rightarrow \begin{aligned}
& \mathcal{O}(4) \otimes V^{1} \\
& \mathcal{O}(2) \otimes V^{3}
\end{aligned} \rightarrow \begin{aligned}
& \mathcal{O}(7) \otimes V^{5} \\
& \mathcal{O}(6) \otimes V^{2} \\
& \mathcal{O}(5) \otimes V^{4}
\end{aligned} \rightarrow \begin{array}{r}
\mathcal{O}(9) \otimes V^{1} \\
\mathcal{O}(8) \otimes V^{3}(7) \otimes V^{5}
\end{array} \rightarrow \cdots \cdots
$$

$n=4:[2]$

$$
\begin{aligned}
& \mathcal{O}(1) \otimes V^{0} \quad \mathcal{O}(3) \otimes V^{1} \quad \mathcal{O}(6) \otimes V^{0} \quad \mathcal{O}(8) \otimes V^{1}
\end{aligned}
$$

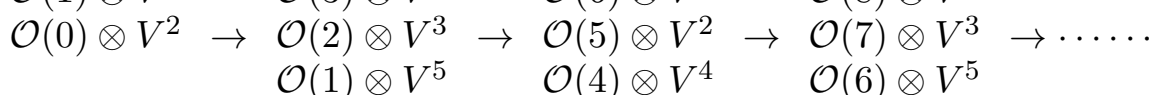

The differential on these complexes is $Q$ from (3.13), whereas before $\eta_{i}$ and $\bar{\eta}_{i}$ act on the exterior algebra $\wedge^{*} V$ in the usual way. It would be interesting to obtain a more intrinsic description of these five objects in $D^{b}\left(X_{\psi}\right)$, to understand their deformations to a general quintic, ${ }^{6}$ to investigate stability at large volume, etc. It is not hard to compute the topological Chern characters of these five objects and to check that they agree with those determined from [61]. For example, the virtual ranks of the objects are given by $(-3,3,-7,8,7)$ for $n=(0,1,2,3,4)$, respectively. The simplest and most canonical object appears to be the one corresponding to $n=3$, namely as found in [67], it carries precisely the topological charges required for anomaly cancellation in a type I (or type IIB orientifold) string compactification on the quintic with non-trivial discrete B-field. (This is mirror to a type IIA orientifold compactification on the mirror quintic.) It is natural therefore to assume that this corresponds to a rank 8 bundle which moreover is stable at large volume on the quintic.

\footnotetext{
${ }^{6}$ We thank Tony Pantev and Ron Donagi for extensive discussions on possible such descriptions.
} 


\subsection{From matrix factorization to curve}

The five complexes in the previous subsection define five objects in $D^{b}\left(X_{\psi}\right)$. (Although semi-infinite, they are quasi-isomorphic to finite complexes because of the eventual periodicity.) As discussed before, to obtain the 625 objects in $D^{b}(Y)$ mirror to the real quintics, we have to make these objects $\left(\mathbb{Z}_{5}\right)^{3}$ equivariant. It would be interesting to understand this construction in detail and, in particular, what happens under the resolution of the orbifold singularities. For our purposes, however, we do not need this. In fact, to compute the normal function by the Abel-Jacobi map, we do not even need to distinguish between the five objects on the quintic. Note that the defining semi-infinite complexes differ only in low homological degree by extensions by line bundles, which contribute only trivially to algebraic K-theory and the Abel-Jacobi map. In other words, all the information about the normal function is contained in the 2-periodic part of the complexes, which is nothing but the original matrix factorization! This fact would have allowed us to bypass all the complications associated with the homological Calabi-Yau/LandauGinzburg correspondence. We nevertheless presented the detailed results in the previous subsection, because we feel that they might be of independent interest, for instance, for questions of stability.

In this subsection, we proceed with the computation of the algebraic second Chern classes of $Q_{\epsilon}^{[\chi]}$, where $\epsilon= \pm 1$. Specifically, the domainwall tension of our interest is given by the image under the Abel-Jacobi map of $Q_{+}^{[\chi]}-Q_{-}^{[\chi]}$. Note that this is well defined since, as follows, e.g., from the index theorem (3.19), the topological Chern classes only depend on $\chi$, and not on $\epsilon$, which is the sign of the square root in (3.13). On the other hand, the Abel-Jacobi map is independent of $\chi$, as explained in the previous paragraph.

It does, however, make a difference whether we work on the quintic or its mirror. On the quintic, we can work with the explicit bundle representatives from (3.30). Let

$$
E_{ \pm}=\operatorname{Ker}\left(\mathcal{O}(2) \oplus \mathcal{O}(1)^{10} \oplus \mathcal{O}(0)^{5} \stackrel{Q_{ \pm}}{\longrightarrow} \mathcal{O}(4)^{5} \oplus \mathcal{O}(3)^{10} \oplus \mathcal{O}(2)\right)
$$

In general, for a bundle of rank $r$ with sufficiently many sections, one can determine the second Chern class by choosing $r-1$ generic sections and finding the codimension-2 locus where those sections fail to be linearly independent. Since twisting by $\mathcal{O}(1)$ will alter the image in the Chow group only trivially, we can always arrange for sufficiently many sections by twisting with $\mathcal{O}(n)$ for $n$ large enough. For bundles such as $E_{ \pm}(n)$, we can 
conveniently find sections ${ }^{7}$ by using the 2-periodicity of the complex (3.30) as the image of $Q$ in the previous step. In the case at hand, we select seven columns of the matrix representation of (3.30) and study the ideal generated by the minors of the resulting $7 \times 16$-dimensional matrix.

After some algebra, we find that the second Chern classes can be represented as

$$
c_{2}\left(E_{+}\right)-c_{2}\left(E_{-}\right)=\left[C_{+}-C_{-}\right] \in \mathrm{CH}^{2}\left(X_{\psi}\right),
$$

where $C_{ \pm}$stands for the algebraic curve

$$
C_{ \pm}=\left\{x_{1}+x_{2}=0, x_{3}+x_{4}=0, x_{5}^{2} \pm \sqrt{5 \psi} x_{1} x_{3}=0\right\} \subset X_{\psi} .
$$

Of course, we are really interested in the matrix factorizations and corresponding bundles as objects in $D^{b}(Y)$, where $Y=X_{\psi} /\left(\mathbb{Z}_{5}\right)^{3}$ is the mirror quintic. Their second Chern classes take values in $\mathrm{CH}^{2}(Y)$ and can be described by considering the image of $C_{ \pm}$under the $\left(\mathbb{Z}_{5}\right)^{3}$ orbifold group. We will study this quotient procedure carefully in the following section.

\section{Main computation}

As before, we let $X_{\psi}$ be the one-parameter family of quintics given by (3.9). The intersection of $X_{\psi}$ with the plane $P=\left\{x_{1}+x_{2}=x_{3}+x_{4}=0\right\}$ is a plane curve of degree 5 which is reducible, with three components (see left part of figure 1). One component is the line $x_{5}=0$, the other two are conics $C_{ \pm}$described by (3.34). Obviously, $\left[C_{+}-C_{-}\right]=0 \in H_{2}\left(X_{\psi}\right)$ for all $\psi$, and thus the cycle $C_{+}-C_{-}$defines a normal function $\nu$ for the one-parameter family of quintics $X_{\psi}$. Consequently, we also obtain a pair of curves and a normal function for the mirror quintic $Y$, which we will denote by the same symbols. Now pick a family of three-chains $\Gamma \subset Y$ with $\partial \Gamma=C_{+}-C_{-}$. The domainwall tension or truncated normal function is given by

$$
\mathcal{T}_{B}=\mathcal{T}_{B}(z)=\int_{\Gamma} \hat{\Omega}
$$

where $\hat{\Omega}$ is a particular choice of holomorphic three-form on $Y$, further specified below.

\footnotetext{
${ }^{7}$ This was initially suggested to us by Nick Warner and anticipated also by Duco van Straten.
} 


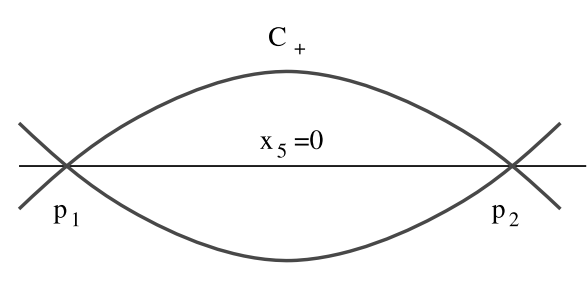

C

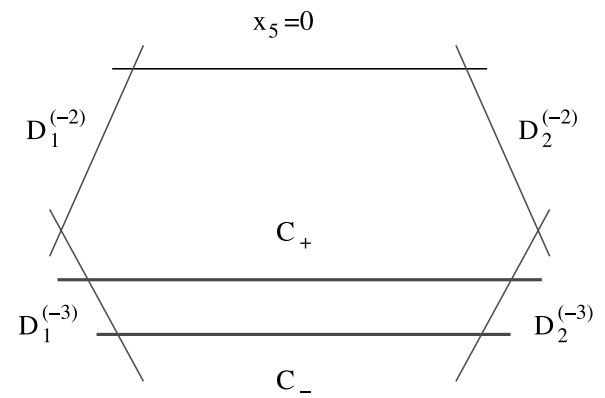

Figure 1: The curves $C_{ \pm}$on $P=\mathbb{C P}^{2}$ (quintic) and on $\widehat{S}=\widehat{\mathbb{C P}^{2} / \mathbb{Z}_{5}}$ (mirror quintic).

The main result of our paper is that the Picard-Fuchs operator from (3.3),

$$
\mathcal{L}=\theta^{4}-5 z(5 \theta+1)(5 \theta+2)(5 \theta+3)(5 \theta+4), \quad \theta=\frac{z d}{\mathrm{~d} z}
$$

acting on $\mathcal{T}_{B}(z)$ gives

$$
\mathcal{L} \mathcal{T}_{B}(z)=\frac{15}{16 \pi^{2}} \sqrt{z}
$$

(as usual, $z=(5 \psi)^{-5}$ ), where the constant is precisely the one in (3.6). We conclude that $\mathcal{T}_{B}(z)$ coincides with $\varpi_{0}(z) \mathcal{T}_{A}(z)$, up to a solution of the homogeneous Picard-Fuchs equation. This is not unexpected since the choice of $\Gamma$ is ambiguous by $H^{3}(Y ; \mathbb{Z})$, so $\mathcal{T}_{B}$ is ambiguous by an integral period. The claim that $\mathcal{T}_{B}(z)-\varpi_{0}(z) \mathcal{T}_{A}(z)$ is indeed an integral period will follow from the analytic continuation performed in [19] and the boundary conditions on $\mathcal{T}_{B}$ as we shall discuss below.

\subsection{Sketch of computation}

The strategy for proving (4.3) is to use the representation of the holomorphic three-form on the hypersurface $\{W=0\}$ as the residue of a meromorphic four-form $\tilde{\Omega}$ on projective space (Griffiths-Dwork method). The domainwall tension, which is defined by integrating the holomorphic three-form over a three-chain $\Gamma$ in $Y$ with $\partial \Gamma=C_{+}-C_{-}$can then be obtained by integrating $\tilde{\Omega}$ over a four-chain which is a tube in $\mathbb{P}^{4} \backslash\{W=0\}$ around $\Gamma{ }^{8}$ By following the usual steps in the derivation of the Picard-Fuchs equation (see, e.g. [5]),

\footnotetext{
${ }^{8}$ We are here temporarily confusing the mirror quintic with the family $X_{\psi}$. The homogeneous Picard-Fuchs equation does not depend on this. The inhomogeneous term, however, does (see below).
} 
the action of $\mathcal{L}$ on the domainwall tension can be reduced to a boundary term consisting of the integral of certain meromorphic three-forms over a tube around the boundary curves $C_{ \pm}$. To be specific, let us consider the contribution from $C_{+}$. The main observation that will make the computation possible is the following.

The curve $C_{+}$lies in the plane $P=\left\{x_{1}+x_{2}=x_{3}+x_{4}=0\right\}$. Therefore, if we could fit the tube around $C_{+}$completely inside $P$, the integral over it of any meromorphic three-form with poles on $W=0$ would vanish. The reason we cannot restrict the computation to $P$ is of course that $P \cap\{W=0\}$ contains not just $C_{+}$, but also $C_{-}$, as well as the line $x_{5}=0$, so that a tube around $C_{+}$inside $P$ will intersect one of the other components, hence $X_{\psi}$. But then, we can fit the tube around $C_{+}$into $P$ except for a small neighborhood of the points where the components of $P \cap\{W=0\}$ meet. There are two such points, $p_{1}=\left\{x_{1}=-x_{2}, x_{3}=x_{4}=x_{5}=0\right\}$ and $p_{2}=\left\{x_{1}=x_{2}=x_{5}=0, x_{3}=-x_{4}\right\}$, and the computation can be localized to a small neighborhood of $p_{1}$ and $p_{2}$, which fit entirely inside an affine patch.

There is, however, an important subtlety in performing this computation as we have just sketched. ${ }^{9}$ Namely, the intersection points $p_{1}$ and $p_{2}$ are actually singular points of the mirror quintic, and these singularities must be resolved first in order to perform the computation. Recall that resolving the singularities amounts to varying the Kähler class on the quintic mirror to a generic value; since the inhomogeneous Picard-Fuchs equation should be independent of the Kähler class, it will not matter how we do the resolution of singularities.

\subsection{Resolution of singularities}

Since the plane $P=\left\{x_{1}+x_{2}=x_{3}+x_{4}=0\right\}$ itself plays an important role in the computation, we also need to resolve singularities that appear on it after passing to the quotient. The symmetry group $\left(\mathbb{Z}_{5}\right)^{3}$ permutes $25 \cdot \frac{5 !}{2 ! 2 !}=$ 750 similar planes, but a $\mathbb{Z}_{5}$ subgroup preserves our plane, with a generator acting via

$$
\left(x_{1},-x_{1}, x_{3},-x_{3}, x_{5}\right) \longmapsto\left(x_{1},-x_{1}, \mathrm{e}^{2 \pi \mathrm{i} / 5} x_{3},-\mathrm{e}^{2 \pi \mathrm{i} / 5} x_{3}, \mathrm{e}^{-4 \pi \mathrm{i} / 5} x_{5}\right) .
$$

This group action has three fixed points, at $p_{1}, p_{2}$, and $(0,0,0,0,1)$, and the first two of these must be resolved. ${ }^{10}$

\footnotetext{
${ }^{9}$ We can attest to the fact that if this subtlety is ignored, a wrong answer is obtained!

${ }^{10}$ The third point does not lie on the quintic mirror for generic $\psi$ and hence need not be resolved.
} 
These singularities on $S=P / \mathbb{Z}_{5}$ are Hirzebruch-Jung singularities $[68,69]$ and can be resolved by classical methods ${ }^{11}$ to obtain a surface $\widehat{S}=\widehat{\mathbb{C P}^{2} / \mathbb{Z}_{5}}$. The result is that each singular point $p_{i}$ is replaced by two rational curves $D_{i}^{(-2)}$ and $D_{i}^{(-3)}$, in the configuration shown in figure 1 . We denote the intersections of (the transforms of) $C_{ \pm}$with the curve $D_{i}^{(-3)}$ by $p_{i, \pm}$.

Resolving the quintic mirror itself is more involved, and we defer the explicit computation to an appendix. The result, however, is the existence of two coordinate charts for the quintic mirror: the first has coordinates

$$
\begin{aligned}
& T=x_{1}^{-1} x_{2}, \\
& X=x_{1} x_{3}^{-2} x_{4}^{3} x_{5}^{-2}, \\
& Y=x_{1}^{-5} x_{5}^{5} \\
& Z=x_{1} x_{3}^{3} x_{4}^{-2} x_{5}^{-2},
\end{aligned}
$$

and the polynomial, locally defining $Y_{\psi}$,

$$
\frac{1}{5}\left(1+T^{5}+X^{2} Y^{2} Z^{3}+X^{3} Y^{2} Z^{2}+Y\right)-\psi T X Y Z
$$

The resolution $\widehat{S}$ of the surface $S$ is given by $T=-1$ and $Z=-X$, and the restriction of the polynomial to $\widehat{S}$ is

$$
\frac{1}{5} Y-\psi X^{2} Y=\frac{1}{5} Y(1+\sqrt{5 \psi} X)(1-\sqrt{5 \psi} X)
$$

The points $p_{1, \pm}$ are given by $X= \pm \frac{1}{\sqrt{5 \psi}}$ and $Y=0$. In the other coordinate chart, we have coordinates

$$
\begin{aligned}
& T^{\prime}=t^{\prime}=x_{3}^{-1} x_{4}, \\
& X^{\prime}=\left(u^{\prime}\right)^{-2}\left(v^{\prime}\right)^{3}\left(w^{\prime}\right)^{-2}=x_{1}^{-2} x_{2}^{3} x_{3} x_{5}^{-2}, \\
& Y^{\prime}=\left(w^{\prime}\right)^{5}=x_{3}^{-5} x_{5}^{5}, \\
& Z^{\prime}=\left(u^{\prime}\right)^{3}\left(v^{\prime}\right)^{-2}\left(w^{\prime}\right)^{-2}=x_{1}^{3} x_{2}^{-2} x_{3} x_{5}^{-2},
\end{aligned}
$$

and polynomial

$$
\frac{1}{5}\left(\left(X^{\prime}\right)^{2}\left(Y^{\prime}\right)^{2}\left(Z^{\prime}\right)^{3}+\left(X^{\prime}\right)^{3}\left(Y^{\prime}\right)^{2}\left(Z^{\prime}\right)^{2} 1+\left(T^{\prime}\right)^{5}++Y^{\prime}\right)-\psi T X Y Z .
$$

\footnotetext{
${ }^{11}$ See [70] for a recent discussion on the physics literature. In fact, the example in figure 2 of [70] is exactly the case we must consider here.
} 
The resolution $\widehat{S}$ of the surface $S$ is given by $T^{\prime}=-1$ and $Z^{\prime}=-X^{\prime}$, and the restriction of the polynomial to $\widehat{S}$ is

$$
\frac{1}{5} Y^{\prime}-\psi\left(X^{\prime}\right)^{2} Y^{\prime}=\frac{1}{5} Y^{\prime}\left(1+\sqrt{5 \psi} X^{\prime}\right)\left(1-\sqrt{5 \psi} X^{\prime}\right) .
$$

The points $p_{2, \pm}$ are given by $X^{\prime}= \pm \frac{1}{\sqrt{5 \psi}}$ and $Y^{\prime}=0$.

\subsection{Inhomogeneous Picard-Fuchs via Griffiths-Dwork}

Let us recall our conventions. We have

$$
W=\frac{1}{5}\left(x_{1}^{5}+x_{2}^{5}+x_{3}^{5}+x_{4}^{5}+x_{5}^{5}\right)-\psi x_{1} x_{2} x_{3} x_{4} x_{5},
$$

and $z=(5 \psi)^{-5}$. To derive the Picard-Fuchs equations by the GriffithsDwork method, we introduce the four-form on $\mathbb{P}^{4}$,

$$
\omega=\sum_{i}(-1)^{i-1} x_{i} \mathrm{~d} x_{1} \wedge \ldots \wedge \widehat{\mathrm{d} x_{i}} \wedge \ldots \wedge \mathrm{d} x_{5}
$$

as well as the contraction of $\omega$ with the tangent vectors $\partial_{i}(i=1, \ldots, 5)$

$$
\omega_{i}=\omega\left(\partial_{i}\right)
$$

A convenient choice of gauge for the holomorphic three-form is

$$
\Omega(z)=\operatorname{Res}_{W=0} \tilde{\Omega}(z), \quad \tilde{\Omega}(z):=\frac{\omega}{W(z)} .
$$

Traditionally, one derives the Picard-Fuchs equation by working with the expression (4.9), thought of as living on the quintic $X_{\psi}$. The holomorphic three-form on the mirror quintic $Y$ can be obtained by pulling back (4.9) in local patches via blowup maps such as described in the appendix. For ordinary periods, the net effect of the quotient by $\left(\mathbb{Z}_{5}\right)^{3}$ is then simply an additional normalization factor of $5^{-3}$ [4]. Such a simple relation is not expected to hold for generic normal functions, so we need to evaluate things more carefully. 
Following the reduction of pole algorithm of Griffiths and keeping track of exact pieces, we find with the above definitions,

$$
\tilde{\mathcal{L}} \tilde{\Omega}:=\left(\left(1-\psi^{5}\right) \partial_{\psi}^{4}-10 \psi^{4} \partial_{\psi}^{3}-25 \psi^{3} \partial_{\psi}^{2}-15 \psi^{2} \partial_{\psi}-1\right) \tilde{\Omega}=-d \tilde{\beta},
$$

where the exact piece is

$$
\begin{aligned}
\tilde{\beta}= & \frac{3 !}{W^{4}}\left(x_{2}^{4} x_{3}^{4} x_{4}^{4} x_{5}^{4} \omega_{1}+\psi x_{2} x_{3}^{5} x_{4}^{5} x_{5}^{5} \omega_{2}+\psi^{2} x_{1} x_{2} x_{3}^{2} x_{4}^{6} x_{5}^{6} \omega_{3}\right. \\
& \left.\quad+\psi^{3} x_{1}^{2} x_{2}^{2} x_{3}^{2} x_{4}^{3} x_{5}^{7} \omega_{4}+\psi^{4} x_{1}^{3} x_{2}^{3} x_{3}^{3} x_{4}^{3} x_{5}^{4} \omega_{5}\right) \\
& +\frac{2}{W^{3}}\left(\psi x_{3} x_{4}^{5} x_{5}^{5} \omega_{3}+3 \psi^{2} x_{1} x_{2} x_{3} x_{4}^{2} x_{5}^{6} \omega_{4}+6 \psi^{3} x_{1}^{2} x_{2}^{2} x_{3}^{2} x_{4}^{2} x_{5}^{3} \omega_{5}\right) \\
& +\frac{1}{W^{2}}\left(\psi x_{4} x_{5}^{5} \omega_{4}+7 \psi^{2} x_{1} x_{2} x_{3} x_{4} x_{5}^{2} \omega_{5}\right) \\
& +\frac{1}{W}\left(\psi x_{5} \omega_{5}\right) .
\end{aligned}
$$

Now the standard Picard-Fuchs operator $\mathcal{L}$ from $(3.3)$ is related to $\tilde{\mathcal{L}}$ from (4.10) by

$$
\mathcal{L}=-\frac{1}{5^{4}} \tilde{\mathcal{L}} \frac{1}{\psi} .
$$

On the other hand, the normalization of the holomorphic three-form in which the solutions (3.4) correspond to primitive integral periods of the mirror quintic is [4]

$$
\hat{\Omega}=\left(\frac{5}{2 \pi \mathrm{i}}\right)^{3} \psi \Omega=\left(\frac{5}{2 \pi \mathrm{i}}\right)^{3} \psi \operatorname{Res}_{W}=0 \frac{\omega}{W} .
$$

The domainwall tension for which we claim (4.3) is defined by

$$
\mathcal{T}_{B}(z)=\int_{\Gamma} \hat{\Omega}(z),
$$

where $\Gamma$ is any three-chain in $Y$ with $\partial \Gamma=C_{+}-C_{-}$. Let $T_{\epsilon}(\Gamma)$ be a small tube around $\Gamma$ of size $\epsilon>0$. Then by (4.9),

$$
\int_{\Gamma} \Omega=\frac{1}{2 \pi \mathrm{i}} \int_{T_{\epsilon}(\Gamma)} \tilde{\Omega}
$$

By combining this with (4.12) and (4.13), the claim (4.3) takes the form

$$
\tilde{\mathcal{L}} \int_{T_{\epsilon}(\Gamma)} \tilde{\Omega}=-\frac{3 \pi^{2}}{5^{1 / 2} \psi^{5 / 2}},
$$

which we now proceed to show. 
There are two types of contributions to the RHS of (4.16), depending on whether the derivatives in $\tilde{\mathcal{L}}$ act on the chain or on $\tilde{\Omega}$. When $\tilde{\mathcal{L}}$ acts entirely on $\tilde{\Omega}$, we use (4.10) and obtain the boundary term

$$
-\int_{T_{\epsilon}\left(C_{+}-C_{-}\right)} \tilde{\beta} .
$$

We will see below that this in fact gives the entire contribution claimed in (4.16). To show that the contributions from derivatives acting on $T_{\epsilon}(\Gamma)$ vanish, we use the fact that as $\psi$ varies, the three-chain $\Gamma$ changes to first order only at its boundary, in a way dictated by the dependence of $C_{ \pm}$on $\psi$. Namely, the first-order variation of $C_{ \pm}$is a section $n \in N_{C_{ \pm} / Y}$ of the normal bundle of $C_{ \pm}$in $Y$. This normal vector lifts to the tube $T_{\epsilon}\left(C_{ \pm}\right)$and we shall show below that for $l=0,1,2,3$,

$$
\int_{T_{\epsilon}\left(C_{+}-C_{-}\right)} \frac{\left(x_{1} x_{2} x_{3} x_{4} x_{5}\right)^{l} \omega(n)}{W^{l+1}}=0
$$

where $\omega(n)$ is the contraction of $\omega$ with the normal vector $n$. Establishing this claim together with the fact that (4.17) evaluates to the RHS of (4.16) will complete the proof.

As described in Section 4.1, we can evaluate integrals of meromorphic three-forms over $T_{\epsilon}\left(C_{ \pm}\right)$as in (4.17) and (4.18), by laying the tube into the plane $P$ (or rather its resolution $\widehat{S}$ ) outside a small neighborhood of the points $p_{i, \pm}$. In those neighborhoods, we can use the coordinates of Section 4.2. Consider $p_{1,+}$, with coordinates (4.4). The curve $C_{+}$is given by $T=-1, X=-Z=1 / \sqrt{5 \psi}$, and locally parametrized by

$$
Y=r \mathrm{e}^{\mathrm{i} \varphi}
$$

varying in a neighborhood of $r=0$. Our tube $T_{\epsilon}\left(C_{+}\right)$is defined by picking a $C^{\infty}$ normal vector $v$ which satisfies $d_{v} W \neq 0$ on $C_{+}$and points inside of $P$ outside of a small neighborhood of $Y=0$. To this end, let $f(r)$ be a non-negative $C^{\infty}$ function with $f(0)=1$ and $f(r)=0$ for $r \geq r_{*}>0$. We then choose

$$
v=\frac{f(r)}{1+(Y / 5)} \partial_{T}-\mathrm{e}^{-\mathrm{i} \varphi} \partial_{X}+\mathrm{e}^{-\mathrm{i} \varphi} \partial_{Z} .
$$

Clearly, $v$ points inside of $P$ for $r>r_{*}$ and one easily checks

$$
\left.d_{v} W\right|_{C_{+}}=f(r)+2 \sqrt{\frac{\psi}{5}} r>0 \text { for } 0 \leq r \leq 2 r_{*} .
$$

(We are here assuming that $\psi>0$. This is no restriction as long as $\psi \neq 0$.) So the part of the tube $T_{\epsilon}\left(C_{+} ; p_{1,+}\right)$ around $C_{+}$which is close to $p_{1,+}$ is 
parametrized as

$$
\begin{gathered}
T=-1+\epsilon \mathrm{e}^{\mathrm{i} \chi} \frac{f(r)}{1+\frac{Y}{5}}, \quad X=-Z=\frac{1}{\sqrt{5 \psi}}-\epsilon \mathrm{e}^{\mathrm{i} \chi} \mathrm{e}^{-\mathrm{i} \varphi}, \\
0 \leq \chi<2 \pi, \quad 0 \leq \varphi<2 \pi, \quad 0 \leq r<2 r_{*} .
\end{gathered}
$$

(In all of this, we should really be taking the limit $\epsilon \rightarrow 0$, but the result will turn out to be independent of $\epsilon$.) There is then a corresponding piece of the tube around $p_{2,+}$. The part of the tube in between does not matter as it lies entirely within $P$, so any meromorphic three-form vanishes there. Finally, the contribution from $C_{-}$will come from substituting $\sqrt{\psi} \rightarrow-\sqrt{\psi}$ in the final answer.

We now apply the coordinate transformation (4.4) to evaluate the threeforms $\omega_{i}$ on the tube (4.22). Choosing $x_{1}=1$, we have

$$
\begin{aligned}
& \omega_{1}=-x_{2} \mathrm{~d} x_{3} \mathrm{~d} x_{4} \mathrm{~d} x_{5}+x_{3} \mathrm{~d} x_{2} \mathrm{~d} x_{4} \mathrm{~d} x_{5}-x_{4} \mathrm{~d} x_{2} \mathrm{~d} x_{3} \mathrm{~d} x_{5}+x_{3} \mathrm{~d} x_{2} \mathrm{~d} x_{3} \mathrm{~d} x_{4}, \\
& \omega_{2}=\mathrm{d} x_{3} \mathrm{~d} x_{4} \mathrm{~d} x_{5}, \\
& \omega_{3}=-\mathrm{d} x_{2} \mathrm{~d} x_{4} \mathrm{~d} x_{5}, \\
& \omega_{4}=\mathrm{d} x_{2} \mathrm{~d} x_{3} \mathrm{~d} x_{5}, \\
& \omega_{5}=-\mathrm{d} x_{2} \mathrm{~d} x_{3} \mathrm{~d} x_{4},
\end{aligned}
$$

and

$$
\begin{aligned}
\frac{\mathrm{d} x_{2}}{x_{2}} & =\frac{\mathrm{d} T}{T}, \\
\frac{\mathrm{d} x_{3}}{x_{3}} & =\frac{3}{5} \frac{\mathrm{d} Z}{Z}+\frac{2}{5} \frac{\mathrm{d} X}{X}+\frac{2}{5} \frac{\mathrm{d} Y}{Y}, \\
\frac{\mathrm{d} x_{4}}{x_{4}} & =\frac{3}{5} \frac{\mathrm{d} X}{X}+\frac{2}{5} \frac{\mathrm{d} Z}{Z}+\frac{2}{5} \frac{\mathrm{d} Y}{Y}, \\
\frac{\mathrm{d} x_{5}}{x_{5}} & =\frac{1}{5} \frac{\mathrm{d} Y}{Y} .
\end{aligned}
$$

After restricting to $X=-Z$, this yields $\omega_{1}=\omega_{2}=\omega_{5}=0$ and

$$
\omega_{3}=\omega_{4}=\mathrm{d} x_{2} \mathrm{~d} x_{3} \mathrm{~d} x_{5}=\frac{x_{2} x_{3} x_{5}}{5 T X Y} \mathrm{~d} T \mathrm{~d} X \mathrm{~d} Y .
$$

Substituting (4.22), we obtain

$$
\mathrm{d} T \mathrm{~d} X \mathrm{~d} Y=\epsilon^{2} \mathrm{e}^{2 \mathrm{i} \chi} \frac{f}{1+(Y / 5)}\left(\frac{r f^{\prime}}{f}-1\right) \mathrm{d} \chi \mathrm{d} \varphi \mathrm{d} r,
$$


where $f^{\prime}=\mathrm{d} f / \mathrm{d} r$. The procedure to compute integrals of the forms $p \mathrm{~d} x_{2} \mathrm{~d} x_{3}$ $\mathrm{d} x_{5} / W^{l+1}$, where $p$ is some monomial in $x_{i}$ 's, over the tube $T_{\epsilon}\left(C_{+} ; p_{1,+}\right)$ is to first write a Laurent series in powers of $\epsilon \mathrm{e}^{\mathrm{i} \chi}$ and $\mathrm{e}^{\mathrm{i} \varphi}$. Integration over $\chi$ and $\varphi$ will then retain only terms of order $\mathrm{e}^{0 \mathrm{i} \chi}$ and $\mathrm{e}^{0 \mathrm{i} \varphi}$, respectively. Finally, we will do the integral over $r$.

To begin with, on the tube we have the expansion

$$
\begin{aligned}
W= & \tilde{\epsilon}\left(f+2 \sqrt{\frac{\psi}{5}} r\right)-\tilde{\epsilon}^{2}\left(2 \tilde{f}^{2}+2 \sqrt{\frac{\psi}{5}} \tilde{f} r+\psi r \mathrm{e}^{-\mathrm{i} \varphi}\right) \\
& +\tilde{\epsilon}^{3}\left(2 \tilde{f}^{3}+\psi \tilde{f} r \mathrm{e}^{-\mathrm{i} \varphi}\right)+\mathcal{O}\left(\tilde{\epsilon}^{4}\right),
\end{aligned}
$$

where $\tilde{\epsilon}=\epsilon \mathrm{e}^{\mathrm{i} \chi}$ and $\tilde{f}=f /(1+(Y / 5))$. In (4.28), we have truncated to order $\epsilon^{3}$ since $\omega_{3} \propto \epsilon^{2}$, and the highest power of $W$ of interest corresponds to $l=3$.

Let us consider the computation of a sample term in $\tilde{\beta}$ from (4.11). Expanding in $\tilde{\epsilon}$, we have

$$
\frac{\psi x_{4} x_{5}^{5} \omega_{4}}{W^{2}}=\left(-\sqrt{\frac{\psi}{5}} \mathrm{e}^{\mathrm{i} \varphi} r \frac{r f^{\prime}-f}{1+(Y / 5)}\left(f+2 \sqrt{\frac{\psi}{5}} r\right)^{-2}+\mathcal{O}(\tilde{\epsilon})\right) \mathrm{d} \chi \mathrm{d} \varphi \mathrm{d} r .
$$

The integration over $\varphi$ clearly kills this term. In fact, it turns out that all the terms in $\tilde{\beta}$ which do not already vanish after restricting to $T_{\epsilon}\left(C_{+} ; p_{1}\right)$ give zero after integration over $\chi$ and $\varphi$.

Going to $p_{2,+}$, where the local coordinates of (4.5) can be accomplished in the above formulas by exchanging $x_{3}$ with $x_{1}$ and $x_{4}$ with $x_{2}$. There are then only two terms to consider.

- The term $6 x_{2}^{4} x_{3}^{4} x_{4}^{4} x_{5}^{4} \omega_{1} / W^{4}$ gives, after integration over $\chi$ and $\varphi$,

$$
(2 \pi)^{2} 12\left(r f^{\prime}-f\right) r^{2}\left[\frac{\psi r^{2}+4 \sqrt{5 \psi} r f+15 f^{2}}{125 \psi(f+2 \sqrt{(\psi / 5)} r)^{6}}\right] .
$$

Integration over $r$ then gives

$$
\frac{3 \pi^{2}}{2 \sqrt{5} \psi^{5 / 2}}
$$

- The term $6 \psi x_{2} x_{3}^{5} x_{4}^{5} x_{5}^{5} \omega_{2} / W^{4}$ gives some complicated expression after integration over the angles, but the integral over $r$ vanishes. 
Taking into account the contribution from $C_{-}$, the final result for (4.17) is

$$
-\int_{T_{\epsilon}\left(C_{+}-C_{-}\right)} \tilde{\beta}=-\frac{3 \pi^{2}}{\sqrt{5} \psi^{5 / 2}},
$$

precisely as claimed.

To show (4.18), we note that the normal vector implementing first-order deformation of $C_{+}$is given by

$$
n=-\frac{x_{5}^{2}}{\sqrt{5 \psi}} \frac{1}{2 \psi} \partial_{3}+\frac{x_{5}^{2}}{\sqrt{5 \psi}} \frac{1}{2 \psi} \partial_{4} .
$$

Thus, we find

$$
\partial_{\psi}^{l} \tilde{\Omega}(n)=l ! \frac{\left(x_{1} x_{2} x_{3} x_{4} x_{5}\right)^{l}}{W^{l+1}} \frac{x_{5}^{2}}{2 \sqrt{5} \psi^{3 / 2}}\left(\omega_{3}-\omega_{4}\right) .
$$

The expression (4.34) vanishes after restriction to the tube, on which $\omega_{3}=\omega_{4}$ holds.

\subsection{Boundary conditions and monodromy}

We have just derived that the domainwall tension of the normal function associated with $C_{+}-C_{-}$satisfies the same inhomogeneous Picard-Fuchs equation (4.3) as the generating function for open Gromov-Witten invariants of the real quintic (3.6). This shows that

$$
\mathcal{T}_{B}(z)=\varpi_{0}(z) \mathcal{T}_{A}(t(z))
$$

up to a solution of the homogeneous Picard-Fuchs equation, i.e., up to a $\mathbb{C}$-linear combination of periods. Identification of the normal function requires equality modulo integral periods, which is a stronger statement. To establish it, we need to determine a sufficient number of boundary conditions on $\mathcal{T}_{B}(z)$. (The boundary conditions on $\mathcal{T}_{A}$ are given by (3.7).)

To fix this result, we make an explicit choice of three-chain connecting $C_{+}$ and $C_{-}$. This is most easily done at $\psi=0$, since $C_{+}$and $C_{-}$degenerate there (see (3.34)). The Landau-Ginzburg monodromy $\psi \rightarrow \mathrm{e}^{2 \pi \mathrm{i} / 5} \psi$ interchanges $C_{+}$with $C_{-}$. The natural choice of three-chain is therefore one that vanishes at $\psi=0$ and changes orientation under the monodromy. 
Now note that in our choice of gauge (4.13), the solutions of the PicardFuchs equation $\mathcal{L} \varpi=0$ actually all vanish as $\psi^{k} \sim z^{-k / 5}$ for some $k=$ $1,2,3,4$ as $\psi \rightarrow 0$. More precisely, the integral periods, known from [4], vanish as $\psi^{1} \sim z^{-1 / 5}$ and are cyclically permuted by the Landau-Ginzburg monodromy $\psi \rightarrow \mathrm{e}^{2 \pi \mathrm{i} / 5} \psi$. We also know, however, that the manifold itself is not singular at $\psi=0$; so none of these vanishing periods corresponds to a vanishing cycle. The integral over the three-chain should therefore vanish faster than any period and just change sign under the monodromy. The unique solution of (4.3) with these properties is given by

$$
\mathcal{T}_{B}(z)=\tau^{\mathrm{orb}}(z)=-\frac{4}{3} \sum_{m=0}^{\infty} \frac{\Gamma(-3 / 2-5 m)}{\Gamma(-3 / 2)} \frac{\Gamma(1 / 2)^{5}}{\Gamma(1 / 2-m)^{5}} z^{-(m+1 / 2)} .
$$

The explicit analytic continuation done in [19] now shows that $\tau^{\text {orb }}(z)$ represents the same solution as $\omega_{0}(z) \mathcal{T}_{A}(t(z))$, up to an integral period that depends on the path chosen to connect $\psi=0$ with $\psi=\infty$.

\section{Summary and conclusions}

In this paper, we have explained why the superpotential/domainwall tension for D-branes wrapped on compact Calabi-Yau manifolds will in general satisfy a differential equation which is an extension of the ordinary PicardFuchs equation. This relationship follows from the insight that certain invariant holomorphic information about the topological D-brane boundary state, as a function of closed string moduli, is contained in the image of the algebraic second Chern class under the Abel-Jacobi map to the intermediate Jacobian, known as Hodge theoretically as a normal function. We have applied this formalism to the B-brane mirror to the real quintic and thereby re-derived the extended Picard-Fuchs equation proposed in [19].

In combination with the proof of the enumerative predictions in the A-model [22], our results put open string mirror symmetry for the real quintic [19] at the same level as the classical mirror theorems of Kontsevich, Givental, Lian-Liu-Yau and others. What is more, we have seen at several places very close connections to ideas from homological mirror symmetry. We have listed in Section 2 several open problems that would make these connections more concrete.

A somewhat unsatisfactory aspect of our derivation is that the nature of the computation in Section 4 was severely analytic. For many reasons, it would be desirable to develop a more algebraic understanding of extended 
Picard-Fuchs equations. The Griffiths infinitesimal invariant is likely to play an important role in such a development. Among other things, this might allow an easier generalization to other situations, especially if the expected connections with the categorical framework can be realized.

\section{Acknowledgments}

We would like to thank Pierre Deligne, Ezra Getzler, Phillip Griffiths, Jaya Iyer, Stefan Müller-Stach, Rahul Pandharipande, Tony Pantev, Duco van Straten, Richard Thomas, and Edward Witten for valuable discussions and communications. We are grateful to the Amsterdam Summer Workshop on String Theory, the Simons Workshop in Mathematics and Physics 2006, the KITP Santa Barbara during the program on String Phenomenology, the Workshop on Homological Mirror Symmetry at IAS, the Workshop on Enumerative Geometry at CRM in Montréal, the Aspen Center for Physics, and the Simons Workshop in Mathematics and Physics 2007, for providing a stimulating atmosphere during various stages of this project, and/or for the opportunity to present some preliminary versions of the results. The work of D.R.M. was supported in part by the NSF grant DMS-0606578. The work of J.W. was supported in part by the Roger Dashen Membership at the Institute for Advanced Study and by the NSF under grant number PHY-0503584. Any opinions, findings, and conclusions or recommendations expressed in this material are those of the authors and do not necessarily reflect the views of the National Science Foundation.

\section{Appendix}

In this section, we describe the resolution of singularities of the quintic mirror, deriving the coordinate charts which are used in making our key computation (see Section 4.3).

The starting point is the singular model of the quintic mirror as a hypersurface inside the singular ambient space $\mathbb{C P}^{4} /\left(\mathbb{Z}_{5}\right)^{3}$. Because the points $p_{1}$ and $p_{2}$ at which we wish to perform our computation are singular points of this quotient, we need to carefully resolve the singularities. We will also explicitly resolve the singularities on the surface $S=\mathbb{C P}^{2} / \mathbb{Z}_{5}$ defined by $x_{1}+x_{2}=0$ and $x_{3}+x_{4}=0$.

A consistent strategy for resolving singularities of the quintic mirror was described in Appendix B of [5]. This strategy involves a choice of blowup, and we will use the choice described in [71] rather than that described in [5]. 
What makes the resolution tricky is that the ambient space $\mathbb{C P}^{4} /\left(\mathbb{Z}_{5}\right)^{3}$ does not have a crepant resolution: the coordinate vertices $(1,0,0,0,0)$ (and cyclic permutations) cannot be resolved without introducing extraneous extra zeros into the holomorphic form of top degree. However, the quintic mirror does not pass through those points, so this fact does not prevent us from resolving the quintic mirror itself.

Each of the points $p_{1}$ and $p_{2}$ lies in the fixed locus of a particular $\left(\mathbb{Z}_{5}\right)^{2}$ subgroup of $\left(\mathbb{Z}_{5}\right)^{3}$. Thus, we will describe a coordinate chart on the blowup for each by describing the blowup of the quotient by the $\left(\mathbb{Z}_{5}\right)^{2}$ subgroup, and indicating how the quotient by the remaining $\mathbb{Z}_{5}$ is to be performed.

The point $p_{1}=(1,-1,0,0,0)$ is contained in the affine chart $x_{1}=1$, and its stabilizer is the $\left(\mathbb{Z}_{5}\right)^{2}$ subgroup of $\left(\mathbb{Z}_{5}\right)^{3}$ which fixes the affine coordinate $x_{2} / x_{1}$.

That is, we begin with affine coordinates $t=x_{2} / x_{1}, u=x_{3} / x_{1}, v=x_{4} / x_{1}$, and $w=x_{5} / x_{1}$ and the $\left(\mathbb{Z}_{5}\right)^{2}$ action on $(u, v, w)$ which preserves the product $u v w$. The rational function invariants under this action are generated by $t$, $u^{5}, v^{5}$, and $u v w$; the remaining $\mathbb{Z}_{5}$ in our full $\left(\mathbb{Z}_{5}\right)^{3}$ symmetry group then preserves $u^{5}$ and $v^{5}$ while acting oppositely on $t$ and on $u v w$, so that the invariants under the full group would include $t^{5}$ and tuvw. The polynomial defining the quintic mirror in this affine coordinate chart is

$$
\frac{1}{5}\left(1+t^{5}+u^{5}+v^{5}+w^{5}\right)-\psi t u v w
$$

and our surface $S$ is defined by $t=-1$ and $v=-u$.

The group action on the surface $S$ is generated by

$$
(u, w) \mapsto\left(\mathrm{e}^{2 \pi \mathrm{i} / 5} u, \mathrm{e}^{-4 \pi \mathrm{i} / 5} w\right),
$$

and the invariant rational monomials for this action are generated by $w^{5}$ and $u w^{-2}$. To describe the corresponding toric geometry, we represent an arbitrary invariant rational monomial in the form

$$
\left(w^{5}\right)^{a}\left(u w^{-2}\right)^{b}=u^{b} w^{5 a-2 b}
$$

and note that the condition for this monomial to be regular, i.e., to have no pole at the origin, $b \geq 0,5 a-2 b \geq 0$. These inequalities determine the toric data: the dual vectors $(0,1)$ and $(5,-2)$ generate a cone consisting of all inequalities satisfied by regular monomials, as depicted in figure 2 (which was borrowed from [70]). 


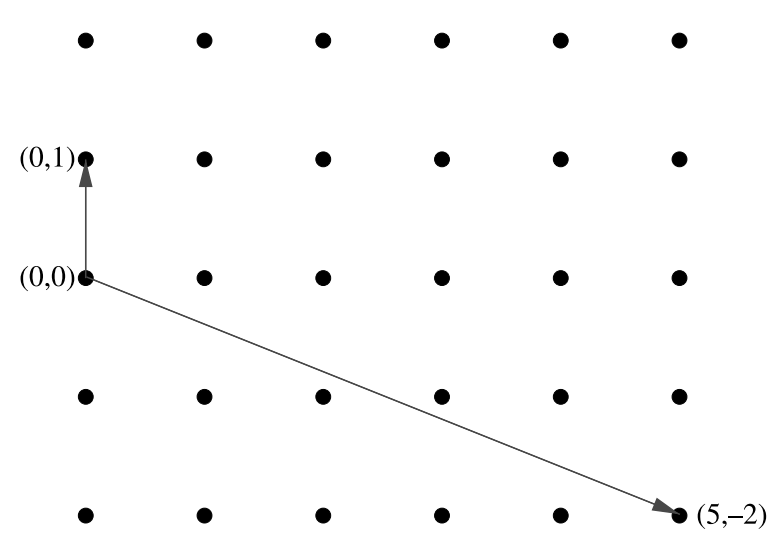

Figure 2: Toric data for the resolution of the $\mathbb{C}^{2} / \mathbb{Z}_{5}$ quotient (from [70]).

To resolve the singularity, we subdivide this cone using cones whose edges form a basis for the lattice $\mathbb{Z}^{2}$. This can always be done by using lattice elements which are close to the origin: in our example, the three subcones are generated by (i) $(0,1)$ and $(1,0)$, (ii) $(1,0)$ and $(3,-1)$, and (iii) $(3,-1)$ and $(5,-2)$.

The coordinates on these three charts have the property that the inequalities defining which monomials are regular within the chart are precisely spanned by the generators of the cone. In our example, the first chart has coordinates

so that

$$
u_{(\mathrm{i})}=u w^{-2}, \quad w_{(\mathrm{i})}=w^{5},
$$

the second chart has coordinates

$$
u^{b} w^{5 a-2 b}=\left(u_{(\mathrm{i})}\right)^{b}\left(w_{(\mathrm{i})}\right)^{a},
$$

$$
u_{(\mathrm{ii})}=u^{3} w^{-1}, \quad w_{(\mathrm{ii})}=w^{2} u^{-1}
$$

so that

and the third chart has coordinates

$$
u^{b} w^{5 a-2 b}=\left(u_{(\mathrm{ii})}\right)^{a}\left(w_{(\mathrm{ii})}\right)^{3 a-b}
$$

$$
u_{(\mathrm{iii})}=u^{5}, \quad w_{(\mathrm{iii})}=w u^{-3},
$$

so that

$$
u^{b} w^{5 a-2 b}=\left(u_{(\mathrm{iii})}\right)^{3 a-b}\left(w_{(\mathrm{iii})}\right)^{5 a-2 b} .
$$

The exceptional curve $D_{1}^{(-3)}$ is represented by $w_{(\mathrm{i})}=u_{(\mathrm{ii})}=0$ and has self-intersection -3 due to the change of coordinate maps

$$
u_{(\mathrm{ii})}=u_{(\mathrm{i})}^{3} w_{(\mathrm{i})}, \quad w_{(\mathrm{ii})}=u_{(\mathrm{i})}^{-1} .
$$


The exceptional curve $D_{1}^{(-2)}$ is represented by $w_{(\mathrm{ii})}=u_{(\mathrm{iii})}=0$ and has self-intersection -2 due to the change of coordinate maps

$$
u_{(\mathrm{iii})}=u_{(\mathrm{ii})}^{2} w_{(\mathrm{ii})}, \quad w_{(\mathrm{iii})}=u_{(\mathrm{ii})}^{-1} .
$$

The defining polynomial for the quintic mirror, when restricted to $S$, takes the following form in these coordinate charts:

$$
\begin{aligned}
\frac{1}{5} w_{(\mathrm{i})}-\psi u_{(\mathrm{i})}^{2} w_{(\mathrm{i})} & =\frac{1}{5} w_{(\mathrm{i})}\left(1-\sqrt{5 \psi} u_{(\mathrm{i})}\right)\left(1+\sqrt{5 \psi} u_{(\mathrm{i})}\right), \\
\frac{1}{5} u_{(\mathrm{ii})} w_{(\mathrm{ii})}^{3}-\psi u_{(\mathrm{ii})} w_{(\mathrm{ii})} & =\frac{1}{5} u_{(\mathrm{ii})} w_{(\mathrm{ii})}\left(w_{(\mathrm{ii})}-\sqrt{5 \psi}\right)\left(w_{(\mathrm{ii})}+\sqrt{5 \psi}\right), \\
\frac{1}{5} u_{(\mathrm{iii})}^{3} w_{(\mathrm{iii})}^{5}-\psi u_{(\mathrm{iii})} w_{(\mathrm{iii})} & =\frac{1}{5} u_{(\mathrm{iii})} w_{(\mathrm{iii})}\left(u_{(\mathrm{iii})} w_{(\mathrm{iii})}^{2}-\sqrt{5 \psi}\right)\left(u_{(\mathrm{iii})} w_{(\mathrm{iii})}^{2}+\sqrt{5 \psi}\right) .
\end{aligned}
$$

Thus, the intersection points $p_{1, \pm}$ of $C_{ \pm}$with $D_{1}^{(-3)}$ can be found in either chart (i) at $\left( \pm(5 \psi)^{-1 / 2}, 0\right)$ or chart (ii) at $\left(0, \pm(5 \psi)^{1 / 2}\right)$. All of these agree with the illustration in figure 1.

We now turn to the resolution of the quintic mirror itself. In order to describe the $\mathbb{C}^{3} /\left(\mathbb{Z}_{5}\right)^{2}$ quotient singularity in terms of toric geometry, we represent an arbitrary invariant rational monomial in the form

$$
\left(u^{5}\right)^{a}\left(v^{5}\right)^{b}(u v w)^{c}=u^{5 a+c} v^{5 b+c} w^{c},
$$

and note that the condition for this monomial to be regular is $5 a+c \geq 0$, $5 b+c \geq 0$, and $c \geq 0$. Those three inequalities determine the toric data: one takes the dual vectors $(5,0,1),(0,5,1),(0,0,1)$ to these inequalities and notes that all inequalities satisfied on the regular functions are non-negative linear combinations of these vectors.

The resolutions of toric geometry are obtained by subdividing the cone generated by those vectors into cones whose generating vectors give a basis for the lattice $\mathbb{Z}^{3}$. There are a number of ways of doing this, but we use the symmetric one illustrated in figure 3 (which is borrowed from [71]). The three dual vectors $(5,0,1),(0,5,1),(0,0,1)$ are the vertices of the large triangle, and the resolution has coordinate charts determined by the small triangles in the diagram.

There are two kinds of coordinate charts. The first type of chart $U_{\alpha \beta}$, labeled by $\alpha$ and $\beta$ with $\alpha \geq 0, \beta \geq 0$, and $\alpha+\beta \leq 4$, corresponds to the upward-pointing triangle in figure 3 with vertices $(\alpha, \beta+1,1),(\alpha, \beta, 1)$, and 


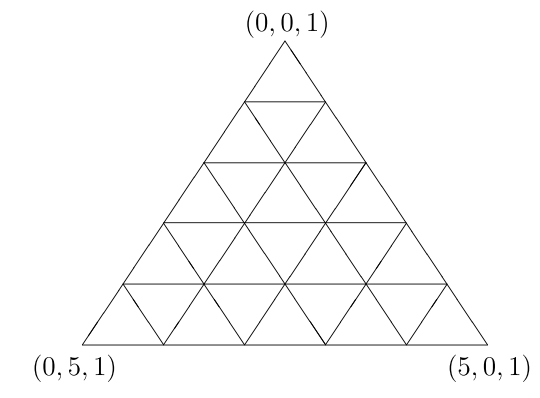

Figure 3: Toric data for the resolution of the $\mathbb{C}^{3} /\left(\mathbb{Z}_{5}\right)^{2}$ quotient (from [71]). $(\alpha+1, \beta, 1)$. This chart will have coordinates $X_{\alpha \beta}, Y_{\alpha \beta}, Z_{\alpha \beta}$, and $T=t$ satisfying

$$
\left(X_{\alpha \beta}\right)^{a \alpha+b(\beta+1)+c}\left(Y_{\alpha \beta}\right)^{a \alpha+b \beta+c}\left(Z_{\alpha \beta}\right)^{a(\alpha+1)+b \beta+c}=\left(u^{5}\right)^{a}\left(v^{5}\right)^{b}(u v w)^{c} .
$$

This can be solved for the coordinates, giving

$$
\begin{aligned}
T & =t \\
X_{\alpha \beta} & =u^{-\beta} v^{5-\beta} w^{-\beta}, \\
Y_{\alpha \beta} & =u^{\alpha+\beta-4} v^{\alpha+\beta-4} w^{\alpha+\beta+1}, \\
Z_{\alpha \beta} & =u^{5-\alpha} v^{-\alpha} w^{-\alpha} .
\end{aligned}
$$

The defining polynomial of the quintic mirror in this chart is

$$
\begin{aligned}
& \frac{1}{5}\left(1+T^{5}+X_{\alpha \beta}^{\alpha} Y_{\alpha \beta}^{\alpha} Z_{\alpha \beta}^{\alpha+1}+X_{\alpha \beta}^{\beta+1} Y_{\alpha \beta}^{\beta} Z_{\alpha \beta}^{\beta}+X_{\alpha \beta}^{4-\alpha-\beta} Y_{\alpha \beta}^{5-\alpha-\beta} Z_{\alpha \beta}^{4-\alpha-\beta}\right) \\
& \quad-\psi T X_{\alpha \beta} Y_{\alpha \beta} Z_{\alpha \beta} .
\end{aligned}
$$

The second type of chart $\widetilde{U}_{\alpha \beta}$, labeled by $\alpha$ and $\beta$ with $\alpha \geq 0, \beta \geq 0$, and $\alpha+\beta \leq 3$, corresponds to the downward-pointing triangles in figure 3 with vertices $(\alpha, \beta+1,1),(\alpha+1, \beta+1,1)$, and $(\alpha+1, \beta, 1)$. This chart will have coordinates $\widetilde{X}_{\alpha \beta}, \widetilde{Y}_{\alpha \beta}, \widetilde{Z}_{\alpha \beta}$, and $\widetilde{T}=t$ satisfying

$$
\left(\widetilde{X}_{\alpha \beta}\right)^{a \alpha+b(\beta+1)+c}\left(\widetilde{Y}_{\alpha \beta}\right)^{a(\alpha+1)+b(\beta+1)+c}\left(\widetilde{Z}_{\alpha \beta}\right)^{a(\alpha+1)+b \beta+c}=\left(u^{5}\right)^{a}\left(v^{5}\right)^{b}(u v w)^{c} .
$$

This can be solved for the coordinates, giving

$$
\begin{aligned}
\widetilde{T} & =t, \\
\widetilde{X}_{\alpha \beta} & =u^{\alpha-4} v^{\alpha+1} w^{\alpha+1},
\end{aligned}
$$




$$
\begin{aligned}
& \widetilde{Y}_{\alpha \beta}=u^{4-\alpha-\beta} v^{4-\alpha-\beta} w^{-1-\alpha-\beta}, \\
& \widetilde{Z}_{\alpha \beta}=u^{\beta+1} v^{\beta-4} w^{\beta+1} .
\end{aligned}
$$

The defining polynomial of the quintic mirror in this chart is

$$
\begin{aligned}
& \frac{1}{5}\left(1+\widetilde{T}^{5}+\widetilde{X}_{\alpha \beta}^{\alpha} \widetilde{Y}_{\alpha \beta}^{\alpha+1} \widetilde{Z}_{\alpha \beta}^{\alpha+1}+\widetilde{X}_{\alpha \beta}^{\beta+1} \widetilde{Y}_{\alpha \beta}^{\beta+1} \widetilde{Z}_{\alpha \beta}^{\beta}+\widetilde{X}_{\alpha \beta}^{4-\alpha-\beta} \widetilde{Y}_{\alpha \beta}^{3-\alpha-\beta} \widetilde{Z}_{\alpha \beta}^{4-\alpha-\beta}\right) \\
& \quad-\psi \widetilde{T} \widetilde{X}_{\alpha \beta} \widetilde{Y}_{\alpha \beta} \widetilde{Z}_{\alpha \beta} .
\end{aligned}
$$

To determine which chart we should use, we restrict the coordinates on $U_{\alpha \beta}$ and $\widetilde{U}_{\alpha \beta}$ to the blowup of $S$ and express them as functions of the coordinates $u_{(i)}$ and $w_{(i)}$ in the first coordinate chart of that blowup. In $U_{\alpha \beta}$ we find

$$
\begin{aligned}
T & =1, \\
X_{\alpha \beta} & =(-1)^{5-\beta} u_{(\mathrm{i})}^{5-2 \beta} w_{(\mathrm{i})}^{2-\beta}, \\
Y_{\alpha \beta} & =(-1)^{\alpha+\beta-4} u_{(\mathrm{i})}^{2 \alpha+2 \beta-8} w_{(\mathrm{i})}^{\alpha+\beta-3}, \\
Z_{\alpha \beta} & =(-1)^{\alpha} u_{(\mathrm{i})}^{5-2 \alpha} w_{(\mathrm{i})}^{2-\alpha},
\end{aligned}
$$

and from this we conclude that $U_{22}$ restricts to this coordinate chart on $S$, and contains the points $p_{1, \pm}$. In $\widetilde{U}_{\alpha \beta}$ we find

$$
\begin{aligned}
\widetilde{T} & =1, \\
\widetilde{X}_{\alpha \beta} & =(-1)^{\alpha+1} u_{(\mathrm{i})}^{2 \alpha-3} w_{(\mathrm{i})}^{\alpha-1}, \\
\widetilde{Y}_{\alpha \beta} & =(-1)^{4-\alpha-\beta} u_{(\mathrm{i})}^{8-2 \alpha-2 \beta} w_{(\mathrm{i})}^{3-\alpha-\beta}, \\
\widetilde{Z}_{\alpha \beta} & =(-1)^{\beta-4} u_{(\mathrm{i})}^{2 \beta-3} w_{(\mathrm{i})}^{\beta-1},
\end{aligned}
$$

and so none of the coordinate charts $\widetilde{U}_{\alpha \beta}$ is a neighborhood of $p_{1, \pm}$.

Thus, the first coordinate chart we use will have coordinates

$$
\begin{aligned}
& T=T_{22}=t=x_{1}^{-1} x_{2}, \\
& X=X_{22}=u^{-2} v^{3} w^{-2}=x_{1} x_{3}^{-2} x_{4}^{3} x_{5}^{-2}, \\
& Y=Y_{22}=w^{5}=x_{1}^{-5} x_{5}^{5}, \\
& Z=Z_{22}=u^{3} v^{-2} w^{-2}=x_{1} x_{3}^{3} x_{4}^{-2} x_{5}^{-2},
\end{aligned}
$$


and polynomial

$$
\frac{1}{5}\left(1+T^{5}+X^{2} Y^{2} Z^{3}+X^{3} Y^{2} Z^{2}+Y\right)-\psi T X Y Z
$$

The resolution $\widehat{S}$ of the surface $S$ is given by $T=-1$ and $Z=-X$, and the restriction of the polynomial to $\widehat{S}$ is

$$
\frac{1}{5} Y-\psi X^{2} Y=\frac{1}{5} Y(1+\sqrt{5 \psi} X)(1-\sqrt{5 \psi} X)
$$

The points $p_{1, \pm}$ are given by $X= \pm 1 / \sqrt{5 \psi}$ and $Y=0$.

To find a neighborhood of the points $p_{2, \pm}$, we use the affine chart $x_{3}=1$ and label the coordinates on this chart as $u^{\prime}=x_{1} / x_{3}, v^{\prime}=x_{2} / x_{3}, t^{\prime}=x_{4} / x_{3}$, and $w^{\prime}=x_{5} / x_{3}$. The defining polynomial becomes

$$
\frac{1}{5}\left(\left(u^{\prime}\right)^{5}+\left(v^{\prime}\right)^{5}+1+\left(t^{\prime}\right)^{5}+\left(w^{\prime}\right)^{5}\right)-\psi t^{\prime} u^{\prime} v^{\prime} w^{\prime}
$$

and the surface $S$ is defined by $t^{\prime}=-1$ and $v^{\prime}=-u^{\prime}$. We have chosen the notation so that the $\left(\mathbb{Z}_{5}\right)^{2}$ subgroup of $\left(\mathbb{Z}_{5}\right)^{3}$ which stabilizes $p_{2}$ acts exactly as in the previous case: acting on $\left(u^{\prime}, v^{\prime}, w^{\prime}\right)$ and preserving the product $u^{\prime} v^{\prime} w^{\prime}$.

Since the combinatorics are identical, the computation produces the same result as in the first part of this appendix. We find a neighborhood of $p_{2, \pm}$ with coordinates

$$
\begin{aligned}
& T^{\prime}=t^{\prime}=x_{3}^{-1} x_{4}, \\
& X^{\prime}=\left(u^{\prime}\right)^{-2}\left(v^{\prime}\right)^{3}\left(w^{\prime}\right)^{-2}=x_{1}^{-2} x_{2}^{3} x_{3} x_{5}^{-2}, \\
& Y^{\prime}=\left(w^{\prime}\right)^{5}=x_{3}^{-5} x_{5}^{5}, \\
& Z^{\prime}=\left(u^{\prime}\right)^{3}\left(v^{\prime}\right)^{-2}\left(w^{\prime}\right)^{-2}=x_{1}^{3} x_{2}^{-2} x_{3} x_{5}^{-2},
\end{aligned}
$$

and polynomial

$$
\frac{1}{5}\left(\left(X^{\prime}\right)^{2}\left(Y^{\prime}\right)^{2}\left(Z^{\prime}\right)^{3}+\left(X^{\prime}\right)^{3}\left(Y^{\prime}\right)^{2}\left(Z^{\prime}\right)^{2} 1+\left(T^{\prime}\right)^{5}++Y^{\prime}\right)-\psi T X Y Z .
$$

The resolution $\widehat{S}$ of the surface $S$ is given by $T^{\prime}=-1$ and $Z^{\prime}=-X^{\prime}$, and the restriction of the polynomial to $\widehat{S}$ is

$$
\frac{1}{5} Y^{\prime}-\psi\left(X^{\prime}\right)^{2} Y^{\prime}=\frac{1}{5} Y^{\prime}\left(1+\sqrt{5 \psi} X^{\prime}\right)\left(1-\sqrt{5 \psi} X^{\prime}\right)
$$

The points $p_{2, \pm}$ are given by $X^{\prime}= \pm 1 / \sqrt{5 \psi}$ and $Y^{\prime}=0$. 
Just for completeness, we include the change of coordinates between these two charts.

$$
\begin{aligned}
T^{\prime} & =\sqrt[5]{\frac{X}{Z}} \\
X^{\prime} & =T^{3} \sqrt[5]{X^{2} Z^{3}} \\
Y^{\prime} & =\frac{1}{\left(X^{2} Y Z^{3}\right)} \\
Z^{\prime} & =T^{-2} \sqrt[5]{X^{2} Z^{3}}
\end{aligned}
$$

These formulas illustrate the important point that the additional $\mathbb{Z}_{5}$ quotient must be considered in each case.

\section{References}

[1] P. Candelas, M. Lynker and R. Schimmrigk, Calabi-Yau manifolds in weighted $\mathbb{P}_{4}$, Nucl. Phys. B 341 (1990), 383-402.

[2] B.R. Greene and M.R. Plesser, Duality in Calabi-Yau moduli space, Nucl. Phys. B 338 (1990), 15-37.

[3] P.S. Aspinwall, C.A. Lütken and G.G. Ross, Construction and couplings of mirror manifolds, Phys. Lett. B 241 (1990), 373-380.

[4] P. Candelas, X.C. De La Ossa, P.S. Green and L. Parkes, A pair of Calabi-Yau manifolds as an exactly soluble superconformal theory, Nucl. Phys. B 359 (1991), 21-74.

[5] D.R. Morrison, Mirror symmetry and rational curves on quintic threefolds: a guide for mathematicians, J. Am. Math. Soc. 6 (1993), 223-247, arXiv:alg-geom/9202004.

[6] A.B. Givental, Equivariant Gromov-Witten invariants, Int. Math. Res. Not. 13 (1996), 613-663, arXiv:alg-geom/9603021.

[7] B.H. Lian, K. Liu and S.-T. Yau, Mirror principle I, Asian J. Math. 1 (1997), 729-763, arXiv:alg-geom/9712011.

[8] D.R. Morrison and M.R. Plesser, Towards mirror symmetry as duality for two-dimensional abelian gauge theories, Nucl. Phys. Proc. Suppl. 46 (1996), 177-186, arXiv:hep-th/9508107.

[9] K. Hori and C. Vafa, Mirror symmetry, arXiv:hep-th/0002222.

[10] E. Witten, Chern-Simons gauge theory as a string theory, Prog. Math. 133 (1995), 637-678, arXiv: hep-th/9207094.

[11] M. Kontsevich, Homological algebra of mirror symmetry, Proc. Int. Congr. Math., Zürich, 1994, Vol. 1, Birkhäuser, 1995, pp. 120-139, arXiv:alg-geom/9411018. 
[12] A. Strominger, S.-T. Yau and E. Zaslow, Mirror symmetry is T-duality, Nucl. Phys. B 479 (1996), 243-259, arXiv: hep-th/9606040.

[13] R. Gopakumar and C. Vafa, M-theory and topological strings I and II, arXiv:hep-th/9809187, arXiv:hep-th/9812127.

[14] M.R. Douglas, D-branes, categories and $N=1$ supersymmetry, J. Math. Phys. 42 (2001), 2818-2843, arXiv: hep-th/0011017.

[15] P.S. Aspinwall and A.E. Lawrence, Derived categories and zero-brane stability, JHEP 08 (2001), 004, arXiv: hep-th/0104147.

[16] A. Kapustin and L. Rozansky, On the relation between open and closed topological strings, Commun. Math. Phys. 252 (2004), 393-414, arXiv: hep-th/0405232.

[17] K. Costello, Topological conformal field theories and Calabi-Yau categories, Adv. Math. 210 (2007), 165-214, arXiv:math.QA/0412149.

[18] A. Caldararu and S. Willerton, The Mukai pairing I, A categorical approach, arXiv:0707.2052 [math.AG].

[19] J. Walcher, Opening mirror symmetry on the quintic, Commun. Math. Phys., 276 (2007), 671-689 arXiv:hep-th/0605162.

[20] G. Almkvist, private communication.

[21] V. Shende, private communication.

[22] R. Pandharipande, J. Solomon and J. Walcher, Disk enumeration on the quintic 3-fold, arXiv:math.SG/0610901.

[23] M. Aganagic and C. Vafa, Mirror symmetry, D-branes and counting holomorphic discs, arXiv:hep-th/0012041.

[24] M. Aganagic, A. Klemm and C. Vafa, Disk instantons, mirror symmetry and the duality web, Z. Naturforsch. A 57 (2002), 1-28, arXiv:hep-th/0105045.

[25] T. Graber and E. Zaslow, Open-string Gromov-Witten invariants: calculations and a mirror "theorem", Orbifolds in mathematics and physics, Contemp. Math., Vol. 310, American Mathematical Society, 2002, pp. 107-121, arXiv:hep-th/0109075.

[26] P. Mayr, Summing up open string instantons and $N=1$ string amplitudes, arXiv:hep-th/0203237.

[27] W. Lerche, P. Mayr and N. Warner, Holomorphic $N=1$ special geometry of open-closed type II strings, arXiv:hep-th/0207259.

[28],$N=1$ special geometry, mixed Hodge variations and toric geometry, arXiv:hep-th/0208039.

[29] S. Govindarajan, T. Jayaraman and T. Sarkar, Disc instantons in linear sigma models, Nucl. Phys. B 646 (2002), 498-523, arXiv:hep-th/0108234. 
[30] B. Forbes, Open string mirror maps from Picard-Fuchs equations, Mirror symmetry, V, AMS/IP Stud. Adv. Math., Vol. 38, American Mathematical Society, 2006, pp. 561-570, arXiv: hep-th/0307167.

[31] E. Witten, Branes and the dynamics of QCD, Nucl. Phys. B 507 (1997), 658-690, arXiv:hep-th/9706109.

[32] P.A. Griffiths, A theorem concerning the differential equations satisfied by normal functions associated to algebraic cycles, Am. J. Math. 101 (1979), 94-131.

[33] P.L. del Angel and S. Müller-Stach, Differential systems associated to families of algebraic cycles, arXiv:math.AG/0305288.

[34] J. Walcher, Extended holomorphic anomaly and loop amplitudes in open topological string, arXiv:hep-th/0705.4098.

[35] P.A. Griffiths, On the periods of certain rational integrals I and II, Ann. Math. 90 (2), (1969), 460-495; ibid. 90 (2), (1969), 496-541.

[36] C.I. Lazaroiu, String field theory and brane superpotentials, JHEP 10 (2001), 018, arXiv:hep-th/0107162.

[37] A. Tomasiello, A-infinity structure and superpotentials, JHEP 09 (2001), 030, arXiv: hep-th/0107195.

[38] M.L. Green, Infinitesimal methods in Hodge theory, Algebraic cycles and Hodge theory, Lecture Notes in Mathematics, Vol. 1594, Springer, 1994, pp. 1-92.

[39] C. Voisin, Hodge theory and complex algebraic geometry II, Cambridge Studies in Advanced Mathematics, Vol. 77, Cambridge University Press, 2003.

[40] - Hodge theory and complex algebraic geometry I, Cambridge Studies in Advanced Mathematics, Vol. 76, Cambridge University Press, 2002.

[41] H. Clemens, Homological equivalence, modulo algebraic equivalence, is not finitely generated, Publ. Math. IHES 58 (1983), 19-38.

[42] C. Voisin, The Griffiths group of a general Calabi-Yau threefold is not finitely generated, Duke Math. J. 102 (2000), 151-186.

[43] E. Witten, New issues in manifolds of SU(3) holonomy, Nucl. Phys. B 268 (1986), 79-112.

[44] C.I. Lazaroiu, D-brane categories, Int. J. Mod. Phys. A 18 (2003), 52995335, arXiv: hep-th/0305095.

[45] S.K. Donaldson and R.P. Thomas, Gauge theory in higher dimensions, The geometric universe, Oxford University Press, 1998, pp. 31-47.

[46] H. Clemens, Cohomology and Obstructions II: Curves on K-trivial Threefolds, arXiv:math.AG/0206219. 
[47] A.N. Tyurin, Nonabelian analogues of Abel's theorem, Izv. Ross. Akad. Nauk Ser. Mat. 65 (2001), 133-196; Izvestija Math. 65 (2001), $123-180$.

[48] C. Vafa, Extending mirror conjecture to Calabi-Yau with bundles, arXiv:hep-th/9804131.

[49] S. Kachru, S. Katz, A.E. Lawrence and J. McGreevy, Open string instantons and superpotentials, Phys. Rev. D 62 (2000), 026001, arXiv:hep-th/9912151.

[50] R. Dijkgraaf and C. Vafa, Matrix models, topological strings, and supersymmetric gauge theories, Nucl. Phys. B 644 (2002), 3-20, arXiv:hep-th/0206255.

[51] A. Grothendieck, La théorie des classes de Chern, Bull. Soc. Math. France 86 (1958), 137-154.

[52] P. Deligne, Local behavior of Hodge structures at infinity, Mirror symmetry, II, AMS/IP Stud. Adv. Math., Vol. 1, American Mathematical Society, 1997, pp. 683-699.

[53] D.R. Morrison, Compactifications of moduli spaces inspired by mirror symmetry, Journées de Géométrie Algébrique d'Orsay, Astérisque, Vol. 218, Société Mathématique de France, 1993, pp. 243-271, arXiv:alg-geom/9304007.

[54] - Mathematical aspects of mirror symmetry, Complex Algebraic Geometry, J. Kollár, ed., IAS/Park City Math. Series, Vol. 3, 1997, pp. 265-340, arXiv:alg-geom/9609021.

[55] K. Fukaya, Y.-G. Oh, H. Ohto and K. Ono, Lagrangian intersection theory - anomaly and obstruction, Kyoto University preprint, 2000.

[56] J.P. Solomon, Intersection theory on the moduli space of holomorphic curves with Lagrangian boundary conditions, arXiv:math.SG/0606429.

[57] J. Walcher, Stability of Landau-Ginzburg branes, J. Math. Phys. 46 (2005), 082305, arXiv:hep-th/0412274.

[58] D. Orlov, Derived categories of coherent sheaves and triangulated categories of singularities, arXiv:math.AG/0503632.

[59] P.S. Aspinwall, The Landau-Ginzburg to Calabi-Yau dictionary for D-branes, arXiv:hep-th/0610209.

[60] M. Herbst, K. Hori and D. Page, Phases of $N=2$ theories in $1+1$ dimensions with boundary, arXiv:hep-th/0803-2045; talks at MSRI Berkeley (March 2006), LMU Munich (July 2006), IAS Princeton (January 2007).

[61] I. Brunner, M.R. Douglas, A.E. Lawrence and C. Romelsberger, D-branes on the quintic, JHEP 08 (2000), 015, arXiv: hep-th/9906200. 
[62] A. Recknagel and V. Schomerus, D-branes in Gepner models, Nucl. Phys. B 531 (1998), 185-225, arXiv:hep-th/9712186.

[63] K. Hori, A. Iqbal and C. Vafa, D-branes and mirror symmetry, arXiv:hep-th/0005247.

[64] K. Hori, Boundary $R G$ flows of $N=2$ minimal models, Mirror symmetry, V, AMS/IP Stud. Adv. Math., Vol. 38, American Mathematical Society, 2006, pp. 381-404, arXiv: hep-th/0401139.

[65] K. Hori and J. Walcher, D-branes from matrix factorizations, Comptes Rendus Physique 5 (2004), 1061-1070, arXiv: hep-th/0409204.

[66] E. Witten, Phases of $N=2$ theories in two dimensions, Nucl. Phys. B 403 (1993) 159-222, arXiv:hep-th/9301042.

[67] I. Brunner, K. Hori, K. Hosomichi and J. Walcher, Orientifolds of Gepner models, JHEP 02 (2007), 001, arXiv:hep-th/0401137.

[68] F. Hirzebruch, Über vierdimensionale Riemannsche Flächen mehrdeutiger analytischer Funktionen von zwei komplexen Veränderlichen, Math. Ann. 126 (1953), 1-22.

[69] H.W.E. Jung, Darstellung der Funktionen eines algebraischen Körpers zweier unabhängiger Veränderlicher $x, y$ in der Umgebung einer Stelle $x=a, y=b, \mathrm{~J}$. Reine Angew. Math. 133 (1908), 289-314.

[70] J.A. Harvey, D. Kutasov, E.J. Martinec and G.W. Moore, Localized tachyons and $R G$ flows, arXiv:hep-th/0111154.

[71] D.R. Morrison, Geometric aspects of mirror symmetry, Mathematics unlimited - 2001 and beyond, Springer, 2001, pp. 899-918, arXiv:math. AG/0007090. 